\title{
Numerical solutions of boundary inverse problems for some elliptic partial differential equations
}

\author{
Suxing Zeng \\ West Virginia University
}

Follow this and additional works at: https://researchrepository.wvu.edu/etd

\section{Recommended Citation}

Zeng, Suxing, "Numerical solutions of boundary inverse problems for some elliptic partial differential equations" (2009). Graduate Theses, Dissertations, and Problem Reports. 4555.

https://researchrepository.wvu.edu/etd/4555

This Dissertation is protected by copyright and/or related rights. It has been brought to you by the The Research Repository @ WVU with permission from the rights-holder(s). You are free to use this Dissertation in any way that is permitted by the copyright and related rights legislation that applies to your use. For other uses you must obtain permission from the rights-holder(s) directly, unless additional rights are indicated by a Creative Commons license in the record and/ or on the work itself. This Dissertation has been accepted for inclusion in WVU Graduate Theses, Dissertations, and Problem Reports collection by an authorized administrator of The Research Repository @ WVU.

For more information, please contact researchrepository@mail.wvu.edu. 


\title{
Numerical Solutions of Boundary Inverse Problems for Some Elliptic Partial Differential Equations
}

\author{
Suxing Zeng \\ Dissertation submitted to \\ Eberly College of Arts and Sciences at \\ West Virginia University \\ in partial fulfillment of the requirements for the degree of \\ Doctor of Philosophy in Mathematics \\ Mary Ann Clarke, Ph.D., Chair \\ Larry Banta, Ph.D. \\ Harvey Diamond, Ph.D. \\ Harumi Hattori, Ph.D. \\ Dening Li, Ph.D.
}

Department of Mathematics

Morgantown, West Virginia

2009 


\section{ABSTRACT \\ Numerical Solutions of Boundary Inverse Problems for Some Elliptic Partial Differential Equations \\ Suxing Zeng}

In this dissertation, we study boundary inverse problems for some elliptic partial differential equations. These are problems arising from quantitative analysis of various nondestructive testing techniques in applications. In such a problem, we are interested in using boundary measurements of the solution to recover either an unknown coefficient function in the boundary condition, or a portion of the boundary, or an unknown interior interface. We first introduce formulations of the boundary value problems into integral equations, then design numerical algorithms for solving each of these inverse problems. Numerical implementation and examples are presented to illustrate the feasibility and effectiveness of the numerical methods.

Keywords: Robin inverse problem, inverse linear source problem, boundary integral equation, Tikhonov regularization, Nyström method.

(Some figures in this dissertation are in color only in the electronic version) 


\section{Acknowledgements}

I sincerely express my appreciation to my thesis advisor, Dr. Weifu Fang, and my official advisor, Dr. Mary Ann Clarke, for their excellent guidance, advice and continuous support and encouragement. At the same time, I would like to thank the other committee members for their assistance during the completion of my thesis.

I would also like to thank the Department of Mathematics at West Virginia University for providing me with an excellent environment for my five-year study here. My thanks also goes to all the professors and friends for their help and support. 


\section{Contents}

1 Introduction $\quad 1$

2 Formulations by Boundary Integral Equations $\quad 6$

2.1 Formulation of the Robin Boundary Value

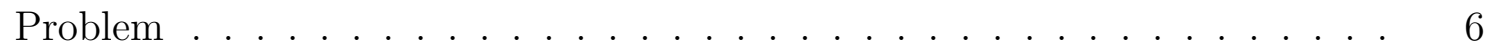

2.2 Formulation of the Linear Source Problem . . . . . . . . . . . . . . 11

2.3 The Nyström's Method with Trigonometric Interpolation . . . . . . . . . . 13

3 Recovery of the Robin Coefficient and the Robin Boundary 18

3.1 Recovery of the Robin Coefficient . . . . . . . . . . . . . . . . . . 18

3.1.1 A Direct Linear Method for the Inverse Problem . . . . . . . . . . . 18

$3.1 .2 \quad$ Numerical Implementation and Examples . . . . . . . . . . . . . . 21

3.2 Recovery of the Unknown Robin Boundary . . . . . . . . . . . . . . 26

3.2.1 The Inverse Problem as a System of Equations . . . . . . . . . . . 26

3.2 .2 Numerical Implementation and Examples . . . . . . . . . . . . . . 31

4 Recovery of the Interface in the Inverse Linear Source Problem 39

4.1 Solution by Least Squares . . . . . . . . . . . . . . . . . . . . . . 39

4.2 The Explicit Forms of the Integral Operators and Their Fréchet Derivatives . 42

4.3 Numerical Implementation and Examples . . . . . . . . . . . . . . . 46

A The Modified Bessel Function $K_{0} \quad 54$ 


\section{List of Figures}

1.1 The cross-sectional model . . . . . . . . . . . . . . . . . 2

1.2 The planar model . . . . . . . . . . . . . . . . . . . . . . . 3

2.1 Exponential decrease of the errors. . . . . . . . . . . . . . . . 17

3.1 Results of 10 recovered $p$ (dashdot) from 10 measurements with same noise level in each plot, for the same true profile $p$ (dashed). . . . . . . . . . . . . 24

3.2 Results of 10 recovered $p$ (dashdot) from 10 measurements with same noise level $\delta=1 \%$, for different true profile $p$ (dashed). . . . . . . . . . . . 25

3.3 Results of recovered $p$ for rounded rectangular domain with various $b$. . . . 26

3.4 Domain setup for the numerical examples. . . . . . . . . . . . . . . . . 31

3.5 Recovery of $\Gamma_{1}$ with noise-free data: from one set of data $u_{0}$ corresponding to input $g$ (left) and from two sets of data $u_{0}$ and $\hat{u}_{0}$ corresponding to inputs $g$

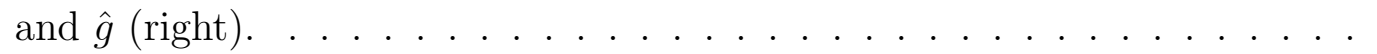

3.6 Recovery of $\Gamma_{1}$ with $1 \%$-noisy data: from one set of data $u_{0}^{\text {(noise) }}$ corresponding to input $g$ (left) and from two sets of data $u_{0}^{\text {(noise) }}$ and $\hat{u}_{0}^{\text {(noise) }}$ corresponding to inputs $g$ and $\hat{g}$ (right). . . . . . . . . . . . . . . . . . . 36

3.7 Recovery of $\Gamma_{1}$ from one set of data corresponding to input $\tilde{g}$ in (3.28): with noise-free data $\tilde{u}_{0}$ (left) and with $1 \%$-noisy data $\tilde{u}_{0}^{\text {(noise) }}$ (right). . . . . . . 38

4.1 Domain setup for the numerical examples. . . . . . . . . . . . . . . 48

4.2 Recovery of different true profiles of $\partial S$ with noise-free data. . . . . . . . . 50

4.3 Recovery of different true profiles of $\partial S$ with $1 \%$-noisy data. . . . . . . . . 51

4.4 Recovery of $\partial S$ with $1 \%$-noisy data on partial $\partial \Omega \ldots$. . . . . . . . . . . 52

4.5 Recovery of $\partial S$ using one set versus two sets of $1 \%$ noisy data. . . . . . . . 53 


\section{Chapter 1}

\section{Introduction}

In this dissertation, we are interested in studying boundary inverse problems for some elliptic partial differential equations (PDE), where boundary measurements of the solution are used to recover unknown coefficients or boundaries in either the boundary conditions or the PDE. These inverse problems originate from the quantitative analysis of many nondestructive testing techniques and evaluations. Typically in such a setting, the desired material profile is represented by a function on an inaccessible portion of the boundary, and measurement of potential corresponding to certain input current is collected on an accessible part of the boundary which is then used to extract the information of the profile, and even to determine the shape and location of the structure. The applications for this type of problem setting abound in engineering and industrial fields. For example, in the evaluation of metal-to-silicon contact quality in semiconductor devices, such as MOSFET (metal-oxide-silicon field-effect transistor), the voltage measurement corresponding to an input current is used to extract information about the contact resistance and the location and shape of the contact window (see e.g. [4, 8, 25, 26]). In the language of thermal imaging, the unknown heat-exchange function can be determined by measuring the temperature on the accessible part of the boundary (see e.g. [1, 3]). In terms of corrosion detection, the material damage profile on the non-accessible part can be recovered by the electrostatic measurement that is made on the accessible part of the boundary (see e.g. $[19,16,5]$ ). There is vast literature on such applications and their analysis and solution methods, and we refer to the above references for more details, discussions and further references.

First, we present a brief description of the models that lead to the inverse problems 
in our study. The original three-dimensional model consists of the Laplace equation for the potential in a three-dimensional region with Robin boundary condition, and it has two reduced two-dimensional models: the cross-sectional model and the thin-layer planar model. It is these two models that we will use to study the related inverse problems.

The cross-sectional model is a two-dimensional version of the original model, and it is an approximation to the original model when the three-dimensional region is relatively long in one direction. Let the two-dimensional domain be $\Omega \subset \mathbf{R}^{2}$ with boundary $\Gamma$, and $U=U(x)$ be the potential. Then the model is (as shown in Figure 1.1):

$$
\begin{cases}\Delta U=0 & \text { in } \quad \Omega, \\ \frac{\partial U}{\partial \nu}+p U=g & \text { on } \quad \Gamma=\partial \Omega,\end{cases}
$$

where $p=p(x) \geq 0$ with support $\Gamma_{1} \subset \Gamma$, and $g=g(x) \geq 0$ is a prescribed input current. The function $p(x)$ and its support $\Gamma_{1}$ represent the desired structural profile (intensity and location). When $p$ and $g$ are given, the problem (1.1) is well-posed and has a solution $U(x)$. This is the usual forward problem.

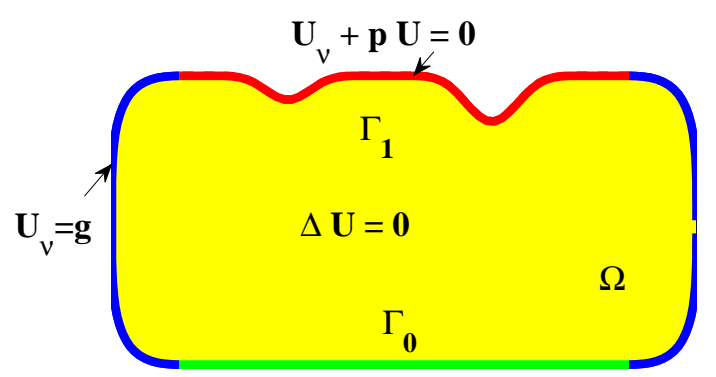

Figure 1.1: The cross-sectional model

Suppose a measurement of $U$ is taken on $\Gamma_{0} \subset \Gamma$, and we wish to use this information to infer information about $p$. This is the inverse problem:

$$
\text { Given } u_{0}=\left.U\right|_{\Gamma_{0}} \text { on } \Gamma_{0} \text {, find } \Gamma_{1} \text { and } p(x) \text { on } \Gamma_{1} \text {. }
$$

When $\Gamma_{1}$ is also given, the inverse problem of finding $p$ from $u_{0}$ is referred to as the Robin inverse problem. It is known that there is no uniqueness in recovering both $\Gamma_{1}$ and $p$ at once (see [6]), while the uniqueness of $p$ alone (assuming $\Gamma_{1}$ given) is a consequence of Holmgreen's theorem $([18])$. 
The planar model is the thin-layer approximation of the three-dimensional model, and it is governed by the following elliptic boundary value problem (as shown in Figure 1.2):

$$
\begin{cases}-\Delta U+p U=0 & \text { in } \quad \Omega \\ \frac{\partial U}{\partial \nu}=g & \text { on } \quad \Gamma,\end{cases}
$$

where $p=p(x) \geq 0$ has support $S \subset \Omega$ and $p=0$ in $\Omega \backslash \bar{S}(\bar{S}$ is the closure of S), and $g$ is prescribed. The desired structural profile is again represented by the function $p(x)$ and its support $S$, except that now $S$ is a region in $\Omega$, not on the boundary. Thus, the inverse problem here is:

$$
\text { Given } u_{0}=\left.U\right|_{\Gamma_{0}} \text { on } \Gamma_{0} \text {, find } S \text { and } p(x) \text { on } S \text {. }
$$

This inverse problem is often referred to as the inverse linear source problem. In general there is no uniqueness in recovering both $p$ and $S$. When $p(x)=p_{0}$ is known, then $S$ is uniquely determined from the extra knowledge of $U$ on $\partial \Omega$ (see [15]).

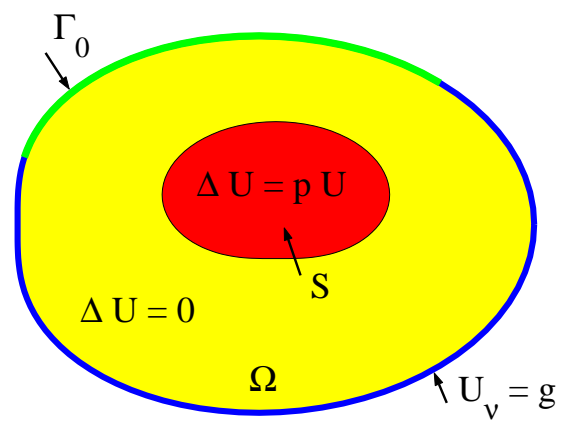

Figure 1.2: The planar model

In Chapter 2, we will present the formulations of the two boundary value problems (1.1) and (1.3) into corresponding boundary integral equations (see e.g. [21, 28]). This approach seems to be natural in view of the fact that the PDEs are relatively simple and available measurements for the inverse problems are boundary measurements of the solutions. For the inverse problems (1.2) to recover the Robin coefficient $p$ and the unknown Robin boundary $\Gamma_{1}$, both the unknown coefficient $p$ with its support $\Gamma_{1}$ and the boundary measurement $u_{0}$ are all on the boundary, and the boundary integral equation nicely captures all these relevant quantities and significantly reduces the size of the computational domain. We will establish 
the equivalence of the integral equation formulation and the boundary value problem (1.1). For the inverse linear source problem (1.4), the formulation involves three integral equations in order to represent the transmission conditions on the interface, and provides a more direct relation between the interface information and the boundary measurements. We will also introduce the Nyström method with trigonometric interpolation for weakly singular integral equations, a numerical method we employ to find numerical solutions for these integral equations.

In Chapter 3, numerical methods for two inverse problems (1.2) of the Robin boundary value problem (1.1) for the Laplacian are presented. The first is the inverse problem of recovering the Robin coefficient on inaccessible boundary from a single partial boundary measurement of the solution. There have been some theoretical and numerical studies for this inverse problem, most of which are based on the PDE model (e.g. $[5,16])$. The integral equation approach was adopted in $[10,24]$ and used to numerically study the inverse problem. In particular, while inverse problems are usually nonlinear and most solution methods are iterative, [24] proposed a linear integral equation approach for the Robin inverse problem, based on the introduction of a new variable. We continue with this approach and present a more direct, much simpler method for recovering the Robin coefficient. The second inverse problem under consideration is to recover part of the Robin boundary from either single or multiple sets of partial boundary measurements. In the literature, there are theoretical and numerical studies for this inverse problem; in particular, the authors in the series of papers $[14,16,17]$ investigated this problem in the PDE setting (1.1) for the case of thin rectangular domains, while the authors in [6] studied a similar problem but in a boundary integral equations setting. We present numerical methods of recovering the unknown boundary portion $\Gamma_{1}$ in an integral equations formulation. We first recast the inverse problem as a direct system of equations, and then solve the nonlinear system in the least-squares sense by iteration using Gauss-Newton directions, with partial regularization. In the case where multiple sets of measurements corresponding to different inputs $g$ are available, we can naturally incorporate the data into this framework to set up algorithms that are more likely to yield better recovery results of the unknown Robin boundary $\Gamma_{1}$.

In Chapter 4, we investigate the inverse problem (1.4) for the recovery of the unknown interface from knowledge of the solution to (1.3) on the outer partial boundary. This problem originates from various industrial applications. For example, it is the planar model in the 
determination of contact resistivity and contact window of electronic devices (e.g.[8]). In heat conduction applications, it represents the problem of recovering the shape and location of an unknown heat source within a bounded region from boundary temperature readings. Another interpretation would be to detect the location of an inhomogeneity in a body from surface measurements of current density and voltage. There have been some theoretical and numerical results for this inverse problem; in particular, [15] studied the problem in the PDE setting to give a uniqueness result, and $[15,9]$ presented Newton-type iterative methods by using the shape derivative with respect to the interface. More recently [29] investigated a similar inverse obstacle problem to recover the unknown obstacle from sets of Cauchy data pairs. Based on the boundary integral equation formulation, we will introduce a numerical method for the inverse problem (1.4), where we seek the solution of a nonlinear least squares problem by Gauss-Newton iteration. As will be seen, the problem becomes increasingly difficult as the size of $\Gamma_{0}$ (where measurement is available) gets smaller. 


\section{Chapter 2}

\section{Formulations by Boundary Integral Equations}

In this Chapter we present the boundary integral equations formulations for the two boundary value problems (1.1) and (1.3). We will depend on these formulations in our numerical study of the related inverse problems (1.2) and (1.4) in the later chapters. In Section 3, we will also present the Nyström method with trigonometric interpolation for numerical solutions of integral equations with weakly singular kernels, which is the numerical method employed in our study.

\subsection{Formulation of the Robin Boundary Value Problem}

Consider the Robin boundary value problem for the Laplace equation in (1.1). Recall that $p=p(x)$ with support $\Gamma_{1} \subset \Gamma$ is the Robin coefficient, and $g=g(x)$ is a prescribed input function, both of which are non-negative functions on $\Gamma$ and have nonempty supports, usually disjoint. Assume that $p \in L^{\infty}(\Gamma)$ and $g \in L^{2}(\Gamma)$.

A weak solution $U$ to (1.1) satisfies

$$
(\triangle U, \phi)=0 \quad \text { for } \quad \phi \in H^{1}(\Omega),
$$


and from Green's identity

$$
\int_{\Omega} \triangle U \phi+\int_{\Omega} \nabla U \cdot \nabla \phi=\int_{\Gamma} \frac{\partial U}{\partial \gamma} \phi
$$

we find

$$
\int_{\Omega} \nabla U \cdot \nabla \phi-\int_{\Gamma} \frac{\partial U}{\partial \gamma} \phi=0
$$

Then from the Robin boundary condition, the weak solution of (1.1) can be defined as $U \in H^{1}(\Omega)$ satisfying

$$
\int_{\Omega} \nabla U \cdot \nabla \phi d x+\int_{\Gamma} p U \phi d s=\int_{\Gamma} g \phi d s \text { for all } \phi \in H^{1}(\Omega) .
$$

The unique existence of such weak solutions can be established by Lax-Milgram Theorem with the help of the trace theorem and a Poincaré-type inequality (see [27]).

Let $\Phi=\Phi(x, y)$ stand for the fundamental solution for the Laplacian in $R^{2}$ :

$$
\Phi(x, y)=\frac{1}{2 \pi} \ln \frac{1}{|x-y|}
$$

for $x, y \in \Omega$ with $x \neq y$. We present two formulations.

Direct formulation. Denote the trace of the solution $U$ to (1.1) on $\Gamma$ by $u \in H^{1 / 2}(\Gamma)$. Then from the third Green identity ([28]), we have:

$$
U(x)=-\int_{\Gamma}\left(\frac{\partial \Phi(x, y)}{\partial \nu_{y}}+p(y) \Phi(x, y)\right) u(y) d s_{y}+\int_{\Gamma} \Phi(x, y) g(y) d s_{y}, \quad x \in \Omega .
$$

Let $x \in \Omega$ approach to the boundary $\Gamma$ and, from jump relations for single and double-layer potentials $([28])$, we find that $u$ satisfies the boundary integral equation:

$$
\frac{1}{2} u(x)+\int_{\Gamma}\left(\frac{\partial \Phi(x, y)}{\partial \nu_{y}}+p(y) \Phi(x, y)\right) u(y) d s_{y}=\int_{\Gamma} \Phi(x, y) g(y) d s_{y}, \quad x \in \Gamma .
$$

In operator form, $(2.4)$ can be written as

$$
\left(\frac{1}{2} \mathcal{I}+\mathcal{D}\right) u+\mathcal{S}(p u)=\mathcal{S} g
$$

with the single and double-layer potential operators defined by

$$
(\mathcal{S} u)(x)=\int_{\Gamma} \Phi(x, y) u(y) d s_{y} \quad \text { and } \quad(\mathcal{D} u)(x)=\int_{\Gamma} \frac{\partial \Phi(x, y)}{\partial \nu_{y}} u(y) d s_{y} \quad \text { for } \quad x \in \Gamma .
$$

Note that the operators have the following mapping properties (e.g. [28]): $\mathcal{S}: H^{-1 / 2}(\Gamma) \rightarrow$ $H^{1 / 2}(\Gamma)$ and $\mathcal{D}: H^{1 / 2}(\Gamma) \rightarrow H^{1 / 2}(\Gamma)$. 
With the direct formulation (2.4) or (2.5), the forward problem for the boundary value problem (1.1) is to find the solution $u$ on $\Gamma$ from (2.5) when functions $p$ and $g$ are given, while the inverse problem is to look for $p$ and its support $\Gamma_{1}$ based on the knowledge of the solution $U$ on the boundary.

Indirect formulation. We can also seek solution $U$ as a single-layer potential:

$$
U(x)=\int_{\Gamma} \Phi(x, y) \varphi(y) d s_{y}, \quad x \in \Omega,
$$

for some potential density $\varphi \in H^{-\frac{1}{2}}(\Gamma)$. As a single-layer potential, $U$ is a harmonic function for any density $\varphi$. In order for $U$ to satisfy the Robin boundary condition, $\varphi$ must solve the following boundary integral equation:

$$
\frac{1}{2} \varphi(x)+\int_{\Gamma} \frac{\partial \Phi(x, y)}{\partial \nu_{x}} \varphi(y) d s_{y}+p(x) \int_{\Gamma} \Phi(x, y) \varphi(y) d s_{y}=g(x), \quad x \in \Gamma .
$$

In operator form, it can be written as

$$
\left(\frac{1}{2} \mathcal{I}+\mathcal{D}^{\prime}\right) \varphi+p \cdot \mathcal{S} \varphi=g
$$

where

$$
\left(\mathcal{D}^{\prime} u\right)(x)=\int_{\Gamma} \frac{\partial \Phi(x, y)}{\partial \nu_{x}} u(y) d s_{y}, \quad x \in \Gamma,
$$

is the dual operator of $\mathcal{D}$. Clearly, $U$ given as the single-layer potential solves (1.1) if and only if its density function $\varphi$ solves the integral equation (2.7). Therefore, the forward problem is solving (2.7) for the density $\varphi$ and then computing $u$ by (2.6), and the inverse problem is to recover $p$ or the unknown boundary $\Gamma_{1}$ from the knowledge of some integrated information about $\varphi$ in (2.7).

Both formulations can be used to solve the solutions of the inverse problems. We note that in [6] the formulation (2.6)-(2.7) is used for the study of completion of Cauchy data for the Laplacian. In our methods for recovering the Robin coefficient $p$ and the unknown boundary $\Gamma_{1}$ that are to be introduced in Chapter 3, we will use the direct formulation for the analysis and numerical computation of the solutions, and the indirect formulation for generating synthetic data $u_{0}$ with the addition of random noise.

In the following, we will establish the equivalence of the boundary integral equation(BIE) formulation (2.4) and the boundary value problem(BVP) (1.1). 
The equivalence of the BIE (2.4) and the BVP (1.1). Similar to the Neumann problem, the Robin problem has a necessary condition for its solution, as stated in the following lemma.

Lemma 2.1 If $u(x) \in L^{2}(\Gamma)$ satisfies the integral equation (2.4), then

$$
\int_{\Gamma} p(x) u(x) d s_{x}=\int_{\Gamma} g(x) d s_{x} .
$$

Proof. From $(2.4)$ we have $\left(\frac{1}{2} \mathcal{I}+\mathcal{D}\right) u=\mathcal{S}(g-p u)$. Apply $\psi$ on both sides of the equation:

$$
\left(\left(\frac{1}{2} \mathcal{I}+\mathcal{D}\right) u, \psi\right)=(\mathcal{S}(g-p u), \psi) \quad \psi \in N\left(\frac{1}{2} I+\mathcal{D}^{\prime}\right) .
$$

Since $\left(\left(\frac{1}{2} \mathcal{I}+\mathcal{D}\right) u, \psi\right)=\left(u,\left(\frac{1}{2} \mathcal{I}+\mathcal{D}^{\prime}\right) \psi\right)=0$, the right hand side of $(2.8)$ is equal to 0 :

$$
\int_{\Gamma} \psi(x) \int_{\Gamma} \Phi(x, y)(g(y)-p(y) u(y)) d s_{y} d s_{x}=0 .
$$

In fact, this is a Fredholm alternative. From [21, theorem 6.25] we know $v(x)=(\mathcal{S} \psi)(x)$ is a solution of the homogeneous interior Neumann problem. By uniqueness, $v(x)$ must be a constant. Changing the orders of integration in (2.8), since $\Phi(x, y)$ is symmetric and $v(x)$ is a constant, $\int_{\Gamma} \Phi(x, y) \psi(x)$ is a constant. Hence $\int_{\Gamma}(g(y)-p(y) u(y)) d s_{y}=0$.

With the help of this lemma, we can state the following theorem:

Theorem 2.2 The boundary value problem (1.1) and the integral equation (2.4) are equivalent.

Proof. If $U$ is the solution to (1.1), obviously, $U$ on $\Gamma$ must satisfy the integral equation (2.4). Conversely, if $u$ satisfies the integral equation (2.4), we can construct a solution $U(x)$ to (1.1) as follows. Define

$$
U(x)=-\int_{\Gamma} \frac{\partial \Phi(x, y)}{\partial \nu_{y}} u(y) d s_{y}+\int_{\Gamma} \Phi(x, y)(g(y)-p(y) u(y)) d s_{y} \quad x \in R^{2} \backslash \Gamma .
$$

Then $U(x)$ is harmonic in $R^{2} \backslash \bar{\Omega}$ and $\Omega$ respectively, and $U \in H^{1}(\Omega)$ and $U \in H^{1}\left(\Omega_{r} \backslash \bar{\Omega}\right)$ for large enough $r\left(\Omega_{r}\right.$ denotes the disk centered at the origin with radius $r$ ) ([28, Theorem 
6.11]). By taking the trace of $U$ on $\Gamma$ from outside $(+)$ and inside $(-)$ of $\Omega$ respectively, and from (2.4) for $u$, we find the traces $U_{+}$and $U_{-}$as:

$$
\begin{array}{rlr}
U_{+}(x) & =-\int_{\Gamma} \frac{\partial \Phi(x, y)}{\partial \nu_{y}} u(y) d s_{y}-\frac{1}{2} u(x)+\int_{\Gamma} \Phi(x, y)(g(y)-p(y) u(y)) d s_{y} \\
& =0 & x \in \Gamma,
\end{array}
$$

and

$$
\begin{array}{rl}
U_{-}(x) & =-\int_{\Gamma} \frac{\partial \Phi(x, y)}{\partial \nu_{y}} u(y) d s_{y}+\frac{1}{2} u(x)+\int_{\Gamma} \Phi(x, y)(g(y)-p(y) u(y)) d s_{y} \\
& =u(x) \\
x & x \Gamma .
\end{array}
$$

From Lemma 2.1, $\int_{\Gamma}\{g(y)-p(y) u(y)\} d s_{y}=0$, hence, $U(x)$ by (2.10) can be also expressed as

$$
\begin{aligned}
U(x)= & -\int_{\Gamma} \frac{\partial \Phi(x, y)}{\partial \nu_{y}} u(y) d s_{y}+\int_{\Gamma} \Phi(x, y)(g(y)-p(y) u(y)) d s_{y} \\
& -\Phi(x, 0) \int_{\Gamma}(g(y)-p(y) u(y)) d s_{y} \quad x \in R^{2} \backslash \bar{\Omega},
\end{aligned}
$$

from which we see that $U(x)$ is bounded in $R^{2} \backslash \bar{\Omega}$. Hence $U(x)$ is harmonic and bounded in $R^{2} \backslash \bar{\Omega}$ with $U_{+}(x)=0$ on $\Gamma$. By the uniqueness of the exterior Dirichlet problem, we have $U(x) \equiv 0$, thus $\frac{\partial U_{+}}{\partial \nu}=0$ on $\Gamma$. By the jump relations of the normal derivatives of single and double-layer potentials on the boundary, we find from (2.10) that:

$$
\begin{aligned}
& \frac{\partial U_{+}}{\partial \nu}(x)=-\frac{\partial^{+}}{\partial \nu} \int_{\Gamma} \frac{\partial \Phi(x, y)}{\partial \nu_{y}} u(y) d s_{y}+\int_{\Gamma} \frac{\partial \Phi(x, y)}{\partial \nu_{x}}(g(y)-p(y) u(y)) d s_{y} \\
&-\frac{1}{2}(g(x)-p(x) u(x)) \\
& x \in \Gamma,
\end{aligned}
$$

and

$$
\begin{aligned}
\frac{\partial U_{-}}{\partial \nu}(x)= & -\frac{\partial^{-}}{\partial \nu} \int_{\Gamma} \frac{\partial \Phi(x, y)}{\partial \nu_{y}} u(y) d s_{y}+\int_{\Gamma} \frac{\partial \Phi(x, y)}{\partial \nu_{x}}(g(y)-p(y) u(y)) d s_{y} \\
& +\frac{1}{2}(g(x)-p(x) u(x)) \\
x & \in \Gamma .
\end{aligned}
$$

Then we have

$$
\frac{\partial U_{-}}{\partial \nu}-\frac{\partial U_{+}}{\partial \nu}=g-p u
$$

Since $\frac{\partial U_{+}}{\partial \nu}=0, \frac{\partial U_{-}}{\partial \nu}=g-p u=g-p U_{-}$. That is, $U(x)$ given in (2.10) on $\Omega$ is indeed the $H^{1}(\Omega)$ solution to $(1.1)$. 


\subsection{Formulation of the Linear Source Problem}

Next, we introduce the boundary integral equations formulation for the linear source problem (1.3), where we assume further that

$$
p(x)=p_{0} \chi_{S}(x)= \begin{cases}p_{0}>0 & \text { if } x \in S \subset \Omega \\ 0 & \text { if } x \in \Omega \backslash S .\end{cases}
$$

The boundary function $g(x)$ is nonnegative on $\partial \Omega$. It is known that, when $\Omega$ and $S$ are smooth domains in $R^{2}$ and $g$ is continuous on $\partial \Omega$, the $H^{1}(\Omega)$ solution $U$ is $C^{1}$-Hölder continuous: $U \in C^{1} 1, \beta(\bar{\Omega})$ (for some $0<\beta<1$ ), and is analytic inside both $S$ and $\Omega \backslash \bar{S}$ (see. e.g. [23]).

Introduce the values of $U$ on $\partial \Omega$ and $\partial S$ as

$$
u(x)=U(x) \quad \text { for } \quad x \in \partial \Omega, \quad v(x)=U(x), \quad w(x)=\frac{\partial U}{\partial \nu}(x) \quad \text { for } \quad x \in \partial S,
$$

where $\nu$ denote the unit outward normal to $\partial \Omega$ or $\partial S$. Notice that, on $\partial S$, both $U$ and its normal derivative $\frac{\partial U}{\partial \nu}$ are continuous. Then the boundary value problem (1.3) for $U$ on $\Omega$ can be transformed into a system of integral equations for $u, v$, and $w$ as follows.

Since $U$ is a harmonic function $\Omega \backslash \bar{S}$, from Green's formula [21, theorem 6.5], we have

$$
\begin{aligned}
U(x)= & \int_{\partial \Omega}\left(\Phi(x, y) g(y)-\frac{\partial \Phi(x, y)}{\partial \nu_{y}} u(y)\right) d s_{y} \\
& -\int_{\partial S}\left(\Phi(x, y) w(y)-\frac{\partial \Phi(x, y)}{\partial \nu_{y}} v(y)\right) d s_{y} \quad \text { for } x \in \Omega \backslash \bar{S}
\end{aligned}
$$

where $\Phi(x, y)$ is the fundamental solution for the Laplacian:

$$
\Phi(x, y)=-\frac{1}{2 \pi} \ln |x-y|
$$

By letting $x \in \Omega \backslash \bar{S}$ approach the boundaries $\partial \Omega$ and $\partial S$, respectively, we can obtain two boundary integral equations:

$$
\begin{aligned}
& \frac{1}{2} u(x)+\int_{\partial \Omega} \frac{\partial \Phi(x, y)}{\partial \nu_{y}} u(y) d s_{y}+\int_{\partial S}\left(\Phi(x, y) w(y)-\frac{\partial \Phi(x, y)}{\partial \nu_{y}} v(y)\right) d s_{y} \\
= & \int_{\partial \Omega} \Phi(x, y) g(y) d s_{y} \quad \text { for } x \in \partial \Omega,
\end{aligned}
$$


and

$$
\begin{aligned}
& \frac{1}{2} v(x)+\int_{\partial \Omega} \frac{\partial \Phi(x, y)}{\partial \nu_{y}} u(y) d s_{y}+\int_{\partial S}\left(\Phi(x, y) w(y)-\frac{\partial \Phi(x, y)}{\partial \nu_{y}} v(y)\right) d s_{y} \\
= & \int_{\partial \Omega} \Phi(x, y) g(y) d s_{y} \quad \text { for } x \in \partial S .
\end{aligned}
$$

Second, $U$ satisfies $-\Delta U+p_{0} U=0$ in $S$, and the fundamental solution associated with this operator is

$$
\Psi(x, y)=\frac{1}{2 \pi} K_{0}\left(q_{0}|x-y|\right)
$$

where $q_{0}=\sqrt{p_{0}}$ and $K_{0}(\cdot)$ denotes the modified Bessel function of order 0 of the second kind. A summary of the relevant special functions is presented in the Appendix. Again, by Green's formula, $U$ in $S$ can be expressed as

$$
U(x)=\int_{\partial S}\left(\Psi(x, y) w(y)-\frac{\partial \Psi(x, y)}{\partial \nu_{y}} v(y)\right) d s_{y} \quad \text { for } x \in S,
$$

and letting $x \in S$ approach $\partial S$ leads to another integral equation on $\partial S$ :

$$
\frac{1}{2} v(x)+\int_{\partial S}\left(\frac{\partial \Psi(x, y)}{\partial \nu_{y}} v(y)-\Psi(x, y) w(y)\right) d s_{y}=0 \quad \text { for } x \in \partial S .
$$

Therefore, solving (1.3) for $U$ in $\Omega$ has been transformed into solving the system of integral equations (2.17), (2.18) and (2.21) for $u$ (on $\partial \Omega$ ), $v$ and $w$ (both on $\partial S$ ).

We can further simplify the system of equations for $(u, v, w)$ by writing it in operator matrix form. To do so, we denote the operators according to the pairing domains of their densities and potentials. For example, denote the single-layer potential operators as

$$
\mathcal{S}_{0}: \partial \Omega \rightarrow \partial \Omega, \quad \mathcal{S}_{1}: \partial S \rightarrow \partial S, \quad \mathcal{S}_{10}: \partial \Omega \rightarrow \partial S, \quad \mathcal{S}_{01}: \partial S \rightarrow \partial \Omega
$$

Similar notations are applied for the double-layer potentials $\mathcal{D}$. The potentials with $\Psi$ are denoted as $\mathcal{S}_{p}$ and $\mathcal{D}_{p}$ (both from $\partial S$ to $\partial S$ ). Then the system consisting of (2.17), (2.18) and (2.21) can be expressed as

$$
\left[\begin{array}{ccc}
\frac{1}{2} \mathcal{I}_{0}+\mathcal{D}_{0} & -\mathcal{D}_{01} & \mathcal{S}_{01} \\
\mathcal{D}_{10} & \frac{1}{2} \mathcal{I}_{1}-\mathcal{D}_{1} & \mathcal{S}_{1} \\
\mathcal{O}_{10} & \frac{1}{2} \mathcal{I}_{1}+\mathcal{D}_{p} & -\mathcal{S}_{p}
\end{array}\right]\left[\begin{array}{c}
u \\
v \\
w
\end{array}\right]=\left[\begin{array}{c}
\mathcal{S}_{0} g \\
\mathcal{S}_{10} g \\
0
\end{array}\right]
$$




\subsection{The Nyström's Method with Trigonometric Inter- polation}

We present briefly the Nyström's method with trigonometric interpolation, a numerical method for numerical solution of integral equations with weakly singular kernels (see [21] for more details).

The Nyström's method is a quadrature method for the approximate solution of integral equations of the second kind with continuous kernels or the kernels with at most a logarithmic singularity. More specifically, the method finds the solution to an integral equation of the second kind

$$
\phi-\mathcal{K} \phi=f
$$

as the approximated solution $\phi_{n}$ of

$$
\phi_{n}-\mathcal{K}_{n} \phi_{n}=f
$$

which reduces to solving a finite-dimensional linear system. Here $\mathcal{K}_{n}$ is the series approximation of the integral operator $\mathcal{K}$ with selected quadrature rules. Here the quadrature rule we choose for the integral operators is trigonometric interpolation. In the following, we use the integral equation (2.4) as an example to demonstrate the Nyström's method with trigonometric interpolation in finding its solution.

To actually perform numerical computations for the boundary integral equations, a parametric description of the boundary is necessary. With the parametrization, we can express the kernels of the integral operators in the formulation (2.4) or (2.6)-(2.7) in explicit forms, which are needed in the numerical calculation of the solutions to the integral equations.

We use a regular 1-periodic parametrization for $\Gamma$ with counterclockwise orientation

$$
x(t)=\left(x_{1}(t), x_{2}(t)\right), \quad 0 \leq t \leq 1
$$

where $x_{1}(t), x_{2}(t) \in C_{p}^{2}[0,1]$ and $\left|x^{\prime}(t)\right| \geq 0$ for $0 \leq t \leq 1$. For $x=\left(x_{1}, x_{2}\right)$, we denote $x^{\perp}=\left(x_{2},-x_{1}\right)$. We also set $u(t)=u(x(t))$ for simplicity. Then the integral operators in 
(2.4) and (2.7) can be expressed explicitly in terms of their kernels as

$$
\begin{aligned}
& (\mathcal{S} u)(t)=\int_{0}^{1} A(t, s) u(s) d s \quad \text { with } \quad A(t, s)=\frac{\left|x^{\prime}(s)\right|}{2 \pi} \ln \frac{1}{|x(t)-x(s)|}, \\
& (\mathcal{D} u)(t)=\int_{0}^{1} B(t, s) u(s) d s \quad \text { and } \quad\left(\mathcal{D}^{\prime} u\right)(t)=\int_{0}^{1} B^{\prime}(t, s) u(s) d s
\end{aligned}
$$

where the kernels $B(t, s)$ and $B^{\prime}(t, s)$ are

$$
B(t, s)=\left\{\begin{array}{ll}
\frac{1}{2 \pi} \frac{x^{\prime}(s)^{\perp} \cdot(x(t)-x(s))}{|x(t)-x(s)|^{2}}, & t \neq s \\
\frac{1}{4 \pi} \frac{x^{\prime}(t)^{\perp} \cdot x^{\prime \prime}(t)}{\left|x^{\prime}(t)\right|^{2}}, & t=s
\end{array} \quad \text { and } \quad B^{\prime}(t, s)=B(s, t) \frac{\left|x^{\prime}(s)\right|}{\left|x^{\prime}(t)\right|}\right.
$$

for $0 \leq t, s \leq 1$. The kernel $A$ is weakly singular while $B$ and $B^{\prime}$ are continuous. The two boundary integral equations (2.4) and (2.7) become

$$
\frac{1}{2} u(t)+\int_{0}^{1}\{B(t, s)+p(s) A(t, s)\} u(s) d s=\int_{0}^{1} A(t, s) g(s) d s
$$

and

$$
\frac{1}{2} \phi(t)+\int_{0}^{1}\left\{B^{\prime}(t, s)+p(t) A(t, s)\right\} \phi(s) d s=g(t),
$$

respectively, for $0 \leq t \leq 1$. When using formulation (2.27), we obtain $u$ from $\phi$ by

$$
u(t)=\int_{0}^{1} A(t, s) \phi(s) d s .
$$

The singularity in the kernel $A(t, s)$ can be rearranged as

$$
\ln |x(t)-x(s)|=\ln (2|\sin (\pi(t-s))|)+A_{0}(t, s)
$$

with continuous kernel

$$
A_{0}(t, s)=\left\{\begin{array}{l}
\ln \frac{|x(t)-x(s)|}{2|\sin (\pi(t-s))|}, \quad t \neq s \\
\ln \frac{\left|x^{\prime}(t)\right|}{2 \pi}, \quad t=s .
\end{array}\right.
$$

So equation (2.4) can be written as:

$$
\frac{1}{2} u(t)+\int_{0}^{1}\left\{A_{1}(t, s) \ln (2|\sin (\pi(t-s))|)+A_{2}(t, s)\right\} u(s) d s=f(t)
$$


for $0 \leq t \leq 1$, where $f(t)=\int_{0}^{1} A(t, s) g(s) d s, A_{1}$ and $A_{2}$ are continuous functions on $[0,1] \times[0,1]$, satisfying periodic conditions such as $A_{1}(t, 0)=A_{1}(t, 1)$. Specifically,

$$
A_{1}(t, s)=-p(s) \frac{\left|x^{\prime}(s)\right|}{2 \pi} \text { and } A_{2}(t, s)=B(t, s)+A_{1}(t, s) A_{0}(t, s) .
$$

Let $[0,1]$ be uniformly partitioned into $2 n$ subintervals with quadrature points $t_{j}=j / 2 n$ for $j=0,1, \cdots, 2 n$. Using the Lagrange basis described in section 11.3 of [21]:

$$
L_{j}(t)=\frac{1}{2 n}\left\{1+2 \sum_{k=1}^{n-1} \cos k\left(2 \pi\left(t-t_{j}\right)\right)+\cos n\left(2 \pi\left(t-t_{j}\right)\right)\right\} \quad n, k \in \mathbf{Z}^{+}
$$

for $t \in[0,1]$ and $j=0, \cdots, 2 n-1$, we replace $A_{1}(t, s) u(s)$ by its trigonometric interpolation polynomials:

$$
A_{1}(t, s) u(s)=\sum_{k=0}^{2 n-1} L_{k}(s) A_{1}\left(t, t_{k}\right) u\left(t_{k}\right) .
$$

Thus equation (2.29) for the approximation $u^{(n)}(t)$ becomes

$$
\frac{1}{2} u(t)+\sum_{k=0}^{2 n-1}\left(R_{k}^{(n)}(t) A_{1}\left(t, t_{k}\right)+\frac{1}{2 n} A_{2}\left(t, t_{k}\right)\right) u\left(t_{k}\right)=f(t)
$$

with

$$
R_{k}^{(n)}(t)=\int_{0}^{1} \ln (2|\sin (\pi(t-s))|) L_{k}(s) d s
$$

for $k=0,1, \cdots, 2 n-1$. By the Nyström's method ([21, Theorem 12.7]), we solve for the values of $u_{j}=u\left(t_{j}\right)$ in the following linear system of equations:

$$
\frac{1}{2} u_{j}+\sum_{k=0}^{2 n-1}\left(R_{|j-k|}^{(n)} A_{1}\left(t_{j}, t_{k}\right)+\frac{1}{2 n} A_{2}\left(t_{j}, t_{k}\right)\right) u\left(t_{k}\right)=f\left(t_{j}\right)
$$

for $j=0,1, \cdots, 2 n-1$. Here $R_{|j-k|}^{(n)}=R_{k}^{(n)}\left(t_{j}\right)$ are determined by the exact integration of (2.31), given by

$$
R_{k}^{(n)}=-\frac{1}{2 n}\left(\sum_{m=1}^{n-1} \frac{1}{m} \cos \frac{m k \pi}{n}+\frac{(-1)^{k}}{2 n}\right)
$$

for $k=0,1, \cdots, 2 n-1$.

Example. We present a test example using the Nyström's method with trigonometric interpolation. For simplicity, take the domain $\Omega$ to be an elliptic region bounded by $\Gamma$ : $x_{1}^{2} / a^{2}+x_{2}^{2} / b^{2}=1$ with $(a, b)=(1,0.2)$. The ellipse has the standard parametrization 
$x=x(t)=(a \cos (2 \pi t), b \sin (2 \pi t))$, for $0 \leq t \leq 1$. We choose the exact solution to the Robin problem (1.1) as $u=a \cos (2 \pi t)+a$, and let $p(x(t))=\sin ^{4}(\pi(t-0.1) / 0.3)$ for $t \in(0.1,0.4)$ and $p=0$ elsewhere. The $g$ on $\Gamma$ is determined by the Robin boundary condition:

$$
g\left(x_{1}, x_{2}\right)=\nabla u\left(x_{1}, x_{2}\right) \cdot \nu+p\left(x_{1}, x_{2}\right) u\left(x_{1}, x_{2}\right) \quad \text { on } \Gamma,
$$

where the outward normal direction vector $\nu$ on $\Gamma$ is given by

$$
\nu=\frac{\left\langle b x_{1} / a, a x_{2} / b\right\rangle}{\sqrt{\left(b x_{1} / a\right)^{2}+\left(a x_{2} / b\right)^{2}}} .
$$

By choosing $g$ this way, we have the exact solution to the integral equation (2.4) as the chosen harmonic function $u$. On the other hand, we find the numerical solution of (2.4) by the Nyström's method. Table 2.1 gives the errors between the exact solution and the numerical solution at $t=0,0.1,0.5$, where the $e_{n}$-column represents the maximum norm of the error vector. The table shows the exponentially decreasing behavior of the errors, as we expect from general error analysis (see $[7, \S 3.5]$ ): there exist positive constants $C$ and $\sigma$ such that

$$
\left|u^{(n)}(t)-u(t)\right| \leq C e^{-\sigma n}, \quad 0 \leq t \leq 2 \pi,
$$

for all $n$. To visualize the behavior, in Figure 2.1, we plot the errors at $t=0.5$ (represented by "+") and the maximum error $e_{n}$ 's (represented by "o") as logarithmic scales for $n=$ $5,10,20,40$. We observe that for different $n$ 's, the data points of "+" and "o" share the same slope, which implies the existence of the constant $\sigma$.

\begin{tabular}{|c|c|c|c|c|}
\hline$n$ & $t=0$ & $t=0.1$ & $t=0.5$ & $e_{n}$ \\
\hline 5 & $9.2066 \mathrm{e}-001$ & $8.2916 \mathrm{e}-001$ & $6.7465 \mathrm{e}-001$ & $9.2066 \mathrm{e}-001$ \\
\hline 10 & $1.0152 \mathrm{e}-002$ & $9.1896 \mathrm{e}-003$ & $6.3795 \mathrm{e}-003$ & $1.0152 \mathrm{e}-002$ \\
\hline 20 & $3.0294 \mathrm{e}-006$ & $2.7423 \mathrm{e}-006$ & $1.9031 \mathrm{e}-006$ & $3.0294 \mathrm{e}-006$ \\
\hline 40 & $2.7267 \mathrm{e}-013$ & $2.4758 \mathrm{e}-013$ & $1.7217 \mathrm{e}-013$ & $2.7800 \mathrm{e}-013$ \\
\hline
\end{tabular}

Table 2.1: Errors between the exact solution and the numerical solution of (2.4). 
CHAPTER 2. FORMULATIONS BY BOUNDARY INTEGRAL EQUATIONS

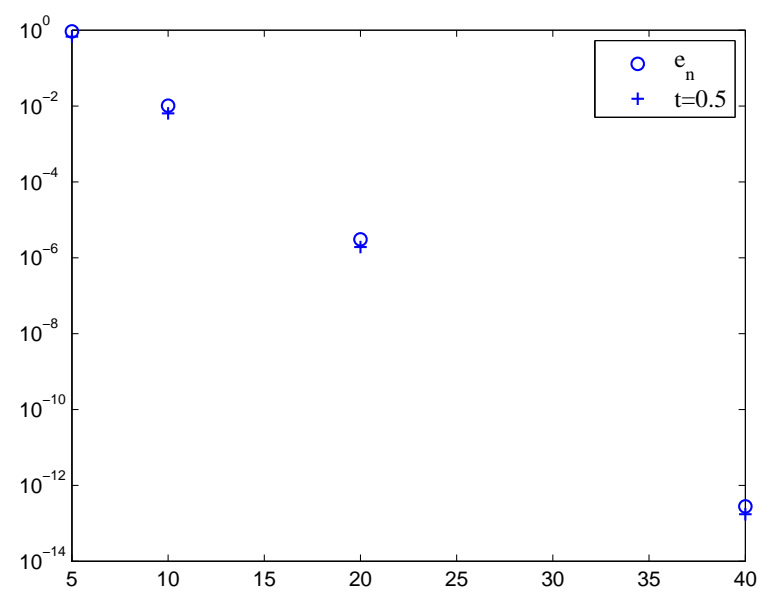

Figure 2.1: Exponential decrease of the errors. 


\section{Chapter 3}

\section{Recovery of the Robin Coefficient and the Robin Boundary}

In this chapter, based on the boundary integral equation formulation introduced in Chapter 2, we study numerical solutions to the two inverse problems of the Robin boundary value problem for the Laplacian in (1.2): one is to recover the Robin coefficient, and the other is to recover the unknown Robin boundary, from the partial boundary measurement of the solution.

\subsection{Recovery of the Robin Coefficient}

The first inverse problem being considered here is: Given $u=u_{0}$ on $\Gamma_{0} \subset \Gamma$ with $\Gamma_{0} \cap \Gamma_{1}=\emptyset$, can we find the Robin coefficient $p$ on $\Gamma_{1}$ ? We present a direct, linear integral equation method for this inverse problem. Numerical examples will be presented to illustrate the effectiveness of this simple yet useful method. Because of its simplicity, it can also be used to provide a quick, quality initial guess for more computationally-expensive iterative algorithms.

\subsubsection{A Direct Linear Method for the Inverse Problem}

Now we introduce the direct solution method for the inverse problem of recovering the coefficient function $p(x)$ on $\Gamma_{1}$ from a single boundary measurement $u_{0}$ of $u$ on $\Gamma_{0}$. 
Direct System for $(\mathbf{u}, \mathbf{v})$. Similar to [24], we introduce a new variable:

$$
v(x)=p(x) u(x) .
$$

The support of $v$ is contained in $\Gamma_{1}$. Then equation (2.5) becomes linear in both $u$ and $v$ :

$$
\left(\frac{1}{2} \mathcal{I}+\mathcal{D}\right) u+\mathcal{S}_{1} v=\mathcal{S} g
$$

where $\left(\mathcal{S}_{1} v\right)(x)=\int_{\Gamma_{1}} \Phi(x, y) v(y) d s_{y}$ for $x \in \Gamma$. Denote the restriction operator from $\Gamma$ to $\Gamma_{0}$ by $\mathcal{R}_{0}: L^{2}(\Gamma) \rightarrow L^{2}\left(\Gamma_{0}\right)$. That is, $\left(\mathcal{R}_{0} u\right)(x)=u(x)$ for $x \in \Gamma_{0}$. Then the measurement of $u$ on $\Gamma_{0}$ can be expressed as:

$$
\mathcal{R}_{0} u=u_{0} .
$$

We cast the inverse problem as a direct problem of finding $p$ from (3.1)-(3.3). Since $u$ on the other part of the boundary is unknown, we will view (3.2)-(3.3) as a system to find both $u$ on $\Gamma$ and $v$ on $\Gamma_{1}$. We write them as a system of operator equations:

$$
\left[\begin{array}{cc}
\frac{1}{2} \mathcal{I}+\mathcal{D} & \mathcal{S}_{1} \\
\mathcal{R}_{0} & \mathcal{O}
\end{array}\right]\left[\begin{array}{l}
u \\
v
\end{array}\right]=\left[\begin{array}{l}
\mathcal{S} g \\
u_{0}
\end{array}\right] \quad \text { or } \quad \mathcal{A} w=f .
$$

Here $\mathcal{O}$ denotes the zero operator from $L^{2}\left(\Gamma_{1}\right)$ to $L^{2}\left(\Gamma_{0}\right)$. Once $u$ on $\Gamma$ and $v$ on $\Gamma_{1}$ are found from (3.4), we can use the simple relation (3.1) to find the Robin coefficient $p$ on $\Gamma_{1}$.

Regularization. The system (3.4) is a linear system for $w=(u, v)^{T}$, but is ill-posed. We will apply the classical Tikhonov regularization method to address the ill-poseness. In fact, we will seek an approximate solution $w_{\alpha}$ to $w$ from the minimization of a quadratic functional that consists of a fidelity term and a regularization term $([21,20])$ :

$$
\min _{w} \frac{1}{2}\|\mathcal{A} w-f\|_{L^{2}[0,1]}^{2}+\frac{\alpha}{2}\left\|w^{\prime}\right\|_{L^{2}[0,1]}^{2}
$$

i.e. $w_{\alpha}$ solves the regularized system

$$
\mathcal{A}^{T} \mathcal{A} w-\alpha \mathcal{H} w=\mathcal{A}^{T} f
$$

where we choose the regularization operator $\mathcal{H}$ as $\mathcal{H}=\left(D_{p}^{2} u, D_{0}^{2} v\right)^{T}\left(D_{p}^{2}\right.$ is the second derivative operator with periodic boundary condition, while $D_{0}^{2}$ is the second derivative operator with zero boundary conditions). The positive constant $\alpha$ is the regularization parameter. In our experiments, the parameter is chosen by inspection and trial and error. 
From (2.1) it can be easily shown that the solution $U \in H^{1}(\Omega)$ is non-negative on $\Omega($ e.g.[27]), and, if the solution is more regular, classical maximum principles can be applied to yield positivity of $u$ on any compact subset of $\Gamma_{1}([5])$. Hence, in such situations, it may be valid to solve $p$ from the relation (3.1) by simple division: $p(x)=v(x) / u(x)$. However, when solving $w_{\alpha}$ from (3.5), the component $u_{\alpha}$ is not guaranteed to be positive, hence we need extra care when computing $p$ from this relation. Based on a Tikhonov regularizaiton consideration for the possibly ill-posed problem of solving $p$ from $p(x) u(x)=v(x)$, we find an approximate solution $p_{\alpha, \beta}(x)$ for the Robin coefficient $p(x)$ as

$$
p_{\alpha, \beta}(x)=\frac{v_{\alpha}^{+}(x) u_{\alpha}^{+}(x)}{\beta+\left(u_{\alpha}^{+}(x)\right)^{2}}, \quad x \in \Gamma_{1},
$$

for some small $\beta \geq 0$, where $v^{+}=\max \{v, 0\}$ denotes the non-negative part of a function $v$. In nearly all of our numerical examples, $u_{\alpha}(x)$ is indeed positive and we are able to set $\beta$ to be 0 . There are other regularization methods for this problem; a common one is to express $p(x)$ in terms of appropriate basis functions such as $B$-splines then find the corresponding coefficients $([6,10])$.

In order to apply the Tikhonov regularization scheme (3.5), we need to establish the injectivity of the operator $\mathcal{A}$ and the denseness of its range.

Theorem 3.1 The operator $\mathcal{A}: L^{2}(\Gamma) \times L^{2}\left(\Gamma_{1}\right) \rightarrow L^{2}(\Gamma) \times L^{2}\left(\Gamma_{0}\right)$ is injective. Furthermore, if the operator $\mathcal{S}$ is injective, then $\mathcal{A}$ has dense range.

Proof. If $\mathcal{A} w=0$ for some $w=(u, v)^{T} \in L^{2}(\Gamma) \times L^{2}\left(\Gamma_{1}\right)$, then

$$
\frac{1}{2} u+\mathcal{D} u+\mathcal{S}_{1} v=0 \text { and } \quad \mathcal{R}_{0} u=0 .
$$

From the first equation, we see that $u$ is the boundary value of the harmonic function in $\Omega$ (also denoted by $u$ for simplicity) with Neumann boundary condition $\frac{\partial u}{\partial \nu}=-\tilde{v}$, where $\tilde{v}$ denotes the zero extension of $v$ on $\Gamma_{1}$ to the entire $\Gamma$. In particular, $\frac{\partial u}{\partial \nu}=0$ on $\Gamma_{0}$ since $\Gamma_{1} \cap \Gamma_{0}=\emptyset$. But the second equation above also gives $u=0$ on $\Gamma_{0}$. Hence, by Holmgren's uniqueness theorem $([18]), u=0$, and consequently $v=0$. Therefore $w=0$ and $\mathcal{A}$ is injective.

To show that $\mathcal{A}$ has dense range, we prove that $\mathcal{A}^{\prime}$ is injective as follows. Note that

$$
\mathcal{A}^{\prime}=\left[\begin{array}{cc}
\frac{1}{2} \mathcal{I}+\mathcal{D}^{\prime} & \mathcal{R}_{0}^{\prime} \\
\mathcal{S}_{1}^{\prime} & \mathcal{O}^{\prime}
\end{array}\right]
$$


where $\mathcal{R}_{0}^{\prime}: L^{2}\left(\Gamma_{0}\right) \rightarrow L^{2}(\Gamma)$ is the zero extension operator from $\Gamma_{0}$ to $\Gamma, \mathcal{S}_{1}^{\prime}: L^{2}(\Gamma) \rightarrow L^{2}\left(\Gamma_{1}\right)$ is $\mathcal{S}$ restricted to $\Gamma_{1}$, and $\mathcal{O}^{\prime}: L^{2}\left(\Gamma_{0}\right) \rightarrow L^{2}\left(\Gamma_{1}\right)$ is the zero operator. If $\mathcal{A}^{\prime} z=0$ for some $z=(\xi, \eta)^{T} \in L^{2}(\Gamma) \times L^{2}\left(\Gamma_{0}\right)$, then

$$
\frac{1}{2} \xi+\mathcal{D}^{\prime} \xi+\mathcal{R}_{0}^{\prime} \eta=0 \quad \text { and } \quad \mathcal{S}_{1}^{\prime} \xi=0 .
$$

From the first equation, the single-layer potential $u=\mathcal{S} \xi$ on $\Omega$ is the solution to the Nuemann boundary value problem with $\frac{\partial u}{\partial \nu}=-\mathcal{R}_{0}^{\prime} \eta$. In particular, $\frac{\partial u}{\partial \nu}=0$ on $\Gamma_{1}$. The second equation above also gives $u=0$ on $\Gamma_{1}$. Hence, by Holmgren's theorem again, we find that $u=0$ and thus $\xi=0$ since $\mathcal{S}$ is injective, consequently $\eta=0$. Therefore $z=(\xi, \eta)^{T}=0$ and $\mathcal{A}^{\prime}$ is injective. Thus $\mathcal{A}^{\prime}$ has dense range.

\subsubsection{Numerical Implementation and Examples}

In this section, we provide implementation details of our direct linear method for finding the solutions to the system (3.4) for $[u, v]^{T}$ or $w$. Numerical results are also presented to show the effectiveness of the method. We adopt the parametrization of boundary $\Gamma$ as in (2.23).

Coefficient matrices. After discretization, using the Nyström's method with trigonometric interpolation, we can find the coefficient matrices for $[u, v]^{T}$ in system (3.4). Divide the interval $[0,1]$ into $2 n$ equal-distance subintervals with nodal points $\left\{t_{j}\right\}_{j=0}^{2 n}\left(t_{0}\right.$ identified with $\left.t_{2 n}\right)$. Suppose the supports for the relevant parts $\Gamma_{1}$ and $\Gamma_{0}$ on $[0,1]$ are $\left[n_{1}, n_{2}\right]$ and $\left[n_{3}, n_{4}\right]$, respectively. Then system (3.4) is reduced to the linear system of equations for the unknown vectors $u=\left[u\left(t_{0}\right), u\left(t_{1}\right), \cdots, u\left(t_{2 n-1}\right)\right]^{T}$ and $v=\left[v\left(t_{n_{1}}\right), v\left(t_{n_{1}+1}\right), \cdots, v\left(t_{n_{2}}\right)\right]^{T}$ :

$$
\left[\begin{array}{cc}
\frac{1}{2} I+D & S_{1} \\
R_{0} & O
\end{array}\right]\left[\begin{array}{l}
u \\
v
\end{array}\right]=\left[\begin{array}{c}
S g \\
u_{0}
\end{array}\right] \quad \text { or } \quad A w=f
$$

where the matrices and vectors are the results of the discretization of the corresponding operators and functions.

By rearranging the logarithmic singularity, the kernel $A(t, s)$ corresponding to the operator $\mathcal{S}$ is expressed as:

$$
A(t, s)=A_{1}(t, s) \ln (2|\sin (\pi(t-s))|)+A_{1}(t, s) A_{0}(t, s)
$$


where

$$
A_{1}=-\frac{\left|x^{\prime}(s)\right|}{2 \pi} \quad \text { and } \quad A_{0}(t, s)=\left\{\begin{array}{l}
\ln \frac{|x(t)-x(s)|}{2|\sin (\pi(t-s))|}, \quad t \neq s \\
\ln \frac{\left|x^{\prime}(t)\right|}{2 \pi}, \quad t=s .
\end{array}\right.
$$

Note that both $A_{0}(t, s)$ and $A_{1}(t, s)$ are continuous kernels. The kernel for the integral operator $\mathcal{D}$ is $B(t, s)$ and it is continuous. Then following the discussion of the Nystöm's method, we find the coefficient matrices of $S$ and $D$ as:

$$
S=\left[S_{j, k}\right] \quad \text { with } \quad S_{j, k}=R_{|j-k|}^{(n)} A_{1}\left(t_{j}, t_{k}\right)+\frac{1}{2 n} A_{1}\left(t_{j}, t_{k}\right) A_{0}\left(t_{j}, t_{k}\right)
$$

and

$$
D=\left[D_{j, k}\right] \quad \text { with } \quad D_{j, k}=\frac{1}{2 n} B\left(t_{j}, t_{k}\right)
$$

for $j, k=0,1, \cdots, 2 n-1$. Here $R_{|j-k|}^{(n)}=R_{k}^{(n)}\left(t_{j}\right)$ are determined by the exact integration of the logarithmic kernels, given by form (2.33). Once the $t$-ranges of $\Gamma_{1}$ and $\Gamma_{0}$ are given, the coefficient matrices $S_{1}$ and $R_{0}$ in (3.7) can be easily determined by $S$ and the identity matrix $I: S_{1}$ consists of the $n_{1}$-th to $n_{2}$-th columns of $S$; while $R_{0}$ is a $\left(n_{4}-n_{3}+1\right) \times 2 n$ zero matrix with the $n_{3}$-th to $n_{4}$-th columns block being replaced by the identity matrix.

We further notice that the discrete system (3.7) has $2 n+\left(n_{4}-n_{3}+1\right)$ equations and $2 n+\left(n_{2}-n_{1}+1\right)$ unknowns, and it is solved by the regularized system (3.5) which has size $\left(2 n+n_{2}-n_{1}+1\right) \times\left(2 n+n_{2}-n_{1}+1\right)$. In this experiment, the regularization operator $\mathcal{H}=\left(D_{p}^{2} u, D_{0}^{2} v\right)$ in system $(3.5)$ are chosen as:

$$
D_{p}^{2}=\left[\begin{array}{ccccc}
-2 & 1 & & & 1 \\
1 & -2 & 1 & & \\
& \ddots & \ddots & \ddots & \\
& & 1 & -2 & 1 \\
1 & & & 1 & -2
\end{array}\right] \text { and } D_{0}^{2}=\left[\begin{array}{ccccc}
-2 & 1 & & & \\
1 & -2 & 1 & & \\
& \ddots & \ddots & \ddots & \\
& & 1 & -2 & 1 \\
& & & 1 & -2
\end{array}\right]
$$

Numerical Examples. In our examples, the domain is chosen as the rounded rectangle:

$$
\Omega=\left\{\left(x_{1}, x_{2}\right): 0 \leq x_{1} \leq a, 0 \leq x_{2} \leq b\right\} \quad \text { with } \quad(a, b)=(1,0.2)
$$

We set the input function $g(t)$ as a characteristic function:

$$
g(t)=1 \quad \text { for } \quad t \in[0.4,0.6] \text { and } g(t)=0 \text { elsewhere. }
$$


The two segments $\Gamma_{1}$ and $\Gamma_{0}$ are

$$
\Gamma_{1}=\{x(t): t \in[0.1,0.4]\} \quad \text { and } \quad \Gamma_{0}=\{x(t): t \in[0.6,0.9]\} \text {. }
$$

Our calculations are carried out using Matlab ${ }^{\circledR}$. The discretization mesh size is set to $h=1 / 200$. To generate the synthetic data $u_{0}$ on $\Gamma_{0}$, we use the indirect formulation (2.6)(2.7) to compose the data $u_{0}(t)$ from $\left.u\right|_{\Gamma_{0}}$ and add to it uniformly distributed random noise of noise level $\delta$ (relative to the $L^{2}$-norm):

$$
u_{0}^{(\text {noise })}(t)=u_{0}(t)+2 \delta\left\|u_{0}(t)\right\|_{2}(\text { rand }-.5),
$$

where rand denotes a random number from the uniform distribution of interval $(0,1)$.

Example 3.2 The effect of noise levels on $p$.

In this example, we investigate how different noise levels in the measurement affect the overall quality of the recovered $p$. The single green dashed line in each plot represents the true $p$ profile and the blue dashed lines are the reconstructions. In order to provide a better illustration, we present in each plot in Figure 3.110 recovered results of $p$ from 10 sets of data within the same noise level; each data is generated by (3.8) with one particular realization of the random noise from $(0,1)$. The regularization parameter $\alpha$ is chosen by experiment. As can be seen from the results, for smaller noise level, the method is capable of recovering the Robin coefficient very well.

Example 3.3 Recovery results for different true $p$ profiles.

In Figure 3.2, we present several recovered results for different true profiles of $p$. Again, in each example, we present 10 times of the results for 10 sets of data with random noise in the same noise level. The true $p$ profiles are the green dotted lines. We observe that the reconstructions for the smooth profiles of true $p$ are generally better than that of the profiles with corners. This is due to our choice of the regularization operator $\mathcal{H}$ as the second derivative operators in system (3.5).

Example 3.4 The effect of the aspect ratio of the domain on the recovery results. 


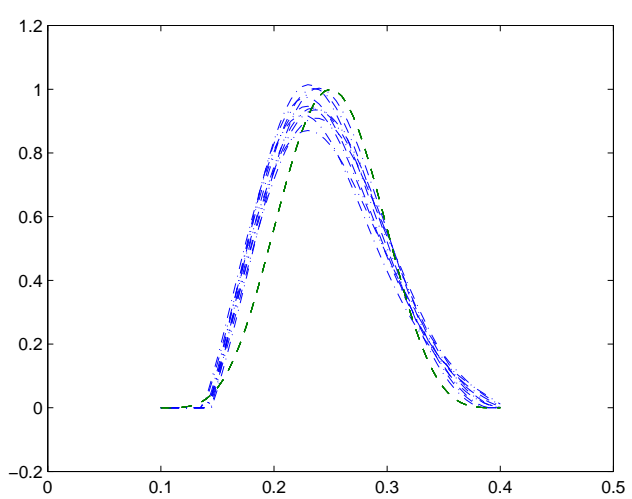

Noise level: $\delta=10 \%$

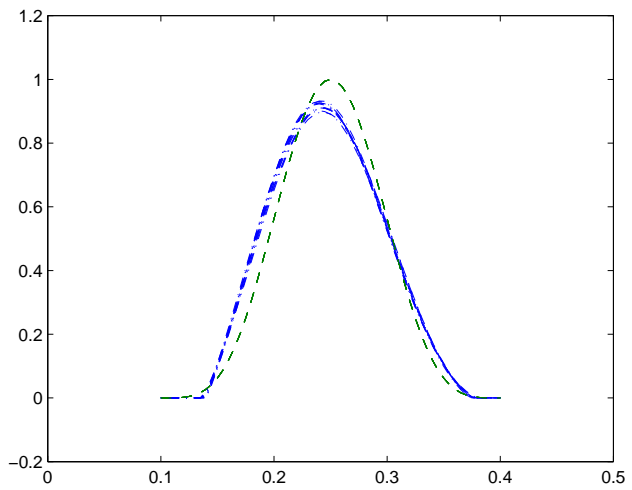

Noise level: $\delta=1 \%$

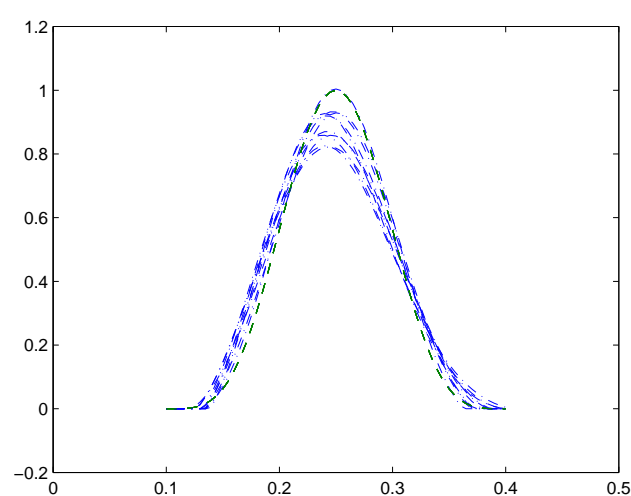

Noise level: $\delta=5 \%$

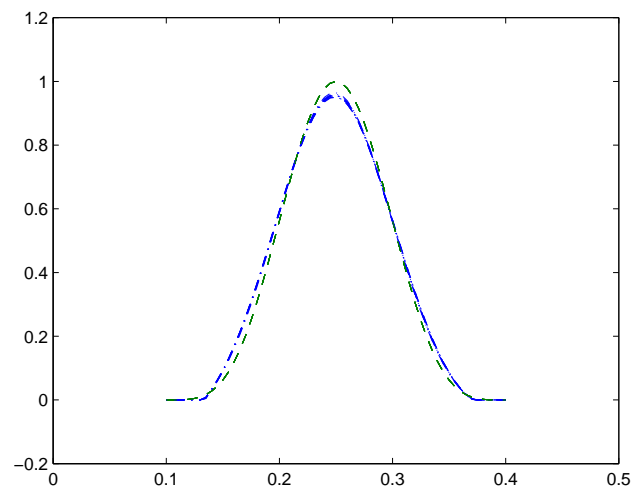

Noise level: $\delta=0.1 \%$

Figure 3.1: Results of 10 recovered $p$ (dashdot) from 10 measurements with same noise level in each plot, for the same true profile $p$ (dashed).

In the last example, we illustrate the effect of the thickness of the rounded rectangular domain on the recovery results. We change the aspect ratio of $a: b$ by fixing $a=1$ and varying $b$ at the numbers $0.2,0.5,1.2$. We use $0.1 \%$-noisy data for the single-hump profile (Figure 3.3, left) and noise-free data for the two-hump profile (Figure 3.3, right). In both cases, as $b$ increases, the recovered $p$ becomes less accurate. This reflects the fact that the problem becomes more ill-posed when $\Gamma_{1}$ is far apart from $\Gamma_{0}$.

Remark. We further remark on the results of our direct method in comparison with the results from methods presented in [24] by similar integral equation formulations. The results by our simple direct method are slightly better in general than the direct least- 


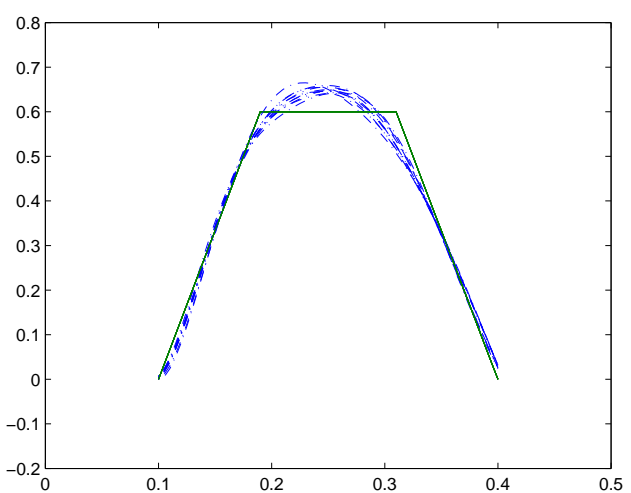

Noise level: $\delta=1 \%$

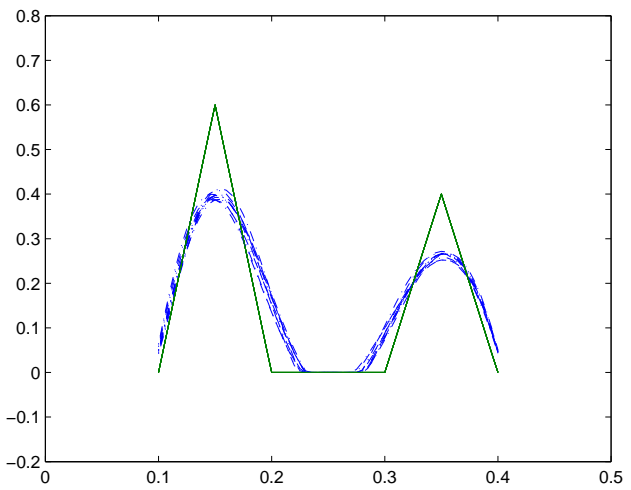

Noise level: $\delta=1 \%$

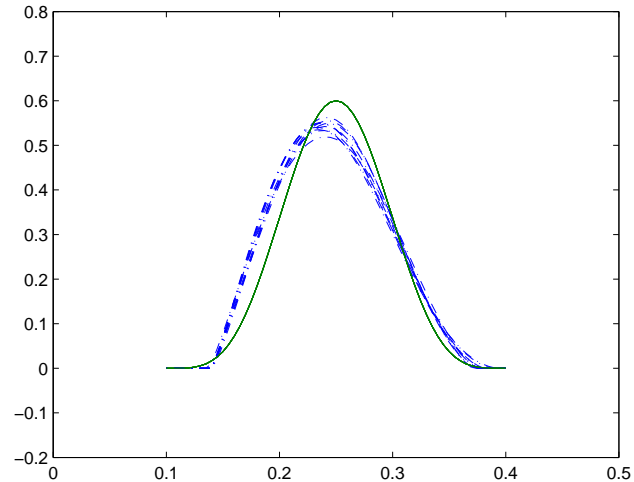

Noise level: $\delta=1 \%$

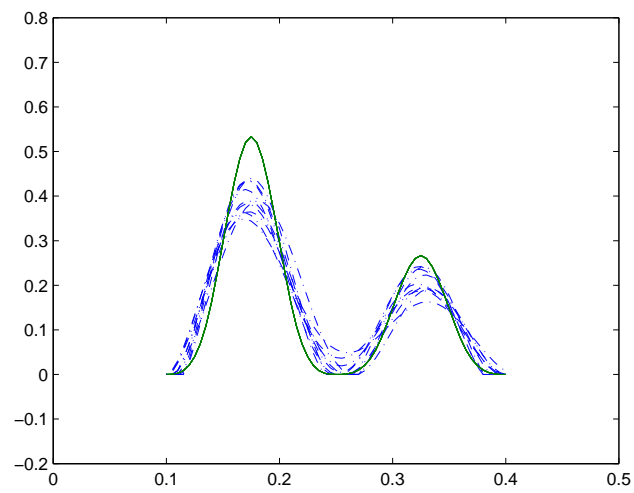

Noise level: $\delta=1 \%$

Figure 3.2: Results of 10 recovered $p$ (dashdot) from 10 measurements with same noise level $\delta=1 \%$, for different true profile $p$ (dashed).

squares method by [24]; the main difference in implementation between the two methods is the size of the linear systems involved: the system (3.5) is twice as large as the size of the normal equation systems for single $v$ in [24]. The iterative quadratic programming method by [24] is more robust and produces better results in general. However, it is worth noting that our simple direct method here is far more economical computationally, yet it is capable of producing comparable results in quality, especially in cases with simple profiles (e.g. Figure 3.1 here vs. Figure 4 in [24]). Because of its simple and economical nature, our direct method here in general can provide a quick quality initial guess for iterative methods that are more computationally extensive, such as the quadratic programming method or 

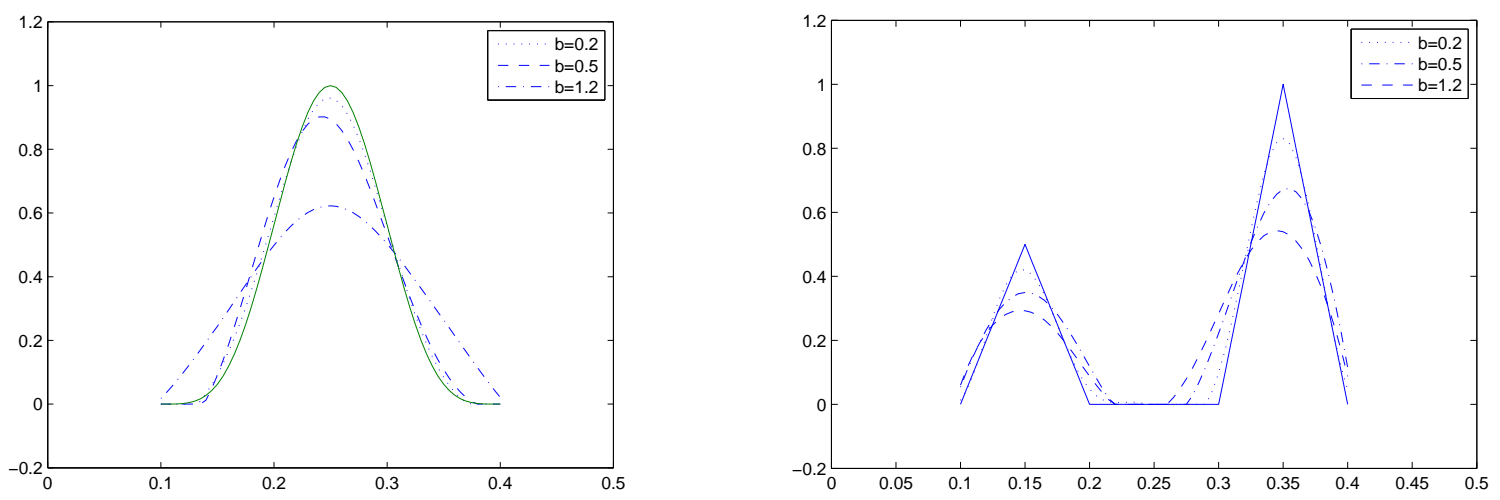

Figure 3.3: Results of recovered $p$ for rounded rectangular domain with various $b$.

methods from a PDE approach.

\subsection{Recovery of the Unknown Robin Boundary}

The second inverse problem we are interested in is to recover the unknown boundary: if the Robin coefficient $p(x)$ has support in $\Gamma_{1} \subset \Gamma$ and $g(x)$ is a prescribed input function, then given $p$ and the measurement $u=u_{0}$ on $\Gamma_{0} \subset \Gamma, \Gamma_{0} \cap \Gamma_{1}=\emptyset$, can we recover the unknown Robin boundary $\Gamma_{1}$ ? In some applications the support of the input $g$ is assumed to be accessible but disjoint from $\Gamma_{0}$, while in other applications it is allowed to have overlap with $\Gamma_{0}$. Based on an integral equations formulation, we present numerical methods for the inverse problem and numerical results to illustrate the effectiveness of our algorithm. We also discuss other factors that might affect the recovery results of the unknown boundary.

\subsubsection{The Inverse Problem as a System of Equations}

Denote the restriction operator from $\Gamma$ to $\Gamma_{0}$ by $\mathcal{R}_{0}$; that is, for $u$ defined on $\Gamma, \mathcal{R}_{0} u$ is defined on $\Gamma_{0}$ with $\left(\mathcal{R}_{0} u\right)(x)=u(x)$ for $x \in \Gamma_{0}$. Then, for a given input $g$, the measurement $u_{0}$ of $u$ on $\Gamma_{0}$ can be expressed as

$$
\mathcal{R}_{0} u=u_{0}
$$

A straightforward approach for the inverse problem would be to recognize the dependence of $u$ on the Robin boundary $\Gamma_{1}$ through (2.4), and find the $\Gamma_{1}$ that "best" fits the data in 
(3.9). That is, we find the solution to the inverse problem as the minimizer of the leastsquares problem

$$
\min _{x}\left\|\mathcal{R}_{0}(u(x))-u_{0}\right\|_{L^{2}[0,1]} .
$$

Note that $u$ depends on $x$ nonlinearly, and one possible approach to solve (3.10) is to use the Gauss-Newton iteration method where the updata step for $x$ is found from the linearization of the equation $\mathcal{R}_{0}(u(x))-u_{0}$. In an alternative approach for this inverse problem, the integrals in (2.4) could be split into integrals on $\Gamma_{0}$ and on $\Gamma \backslash \Gamma_{0}$ :

$$
\begin{array}{r}
\frac{1}{2} u(x)+\int_{\Gamma_{0}} \frac{\partial \Phi(x, y)}{\partial \nu_{y}} u_{0} d s_{y}+\int_{\Gamma \backslash \Gamma_{0}}\left(\frac{\partial \Phi(x, y)}{\partial \nu_{y}}+p(y) \Phi(x, y)\right) u(y) d s_{y} \\
=\int_{\Gamma} \Phi(x, y) g(y) d s_{y}, \quad x \in \Gamma
\end{array}
$$

where $u$ is replaced by the measurement $u_{0}$ on $\Gamma_{0}$; the resulting system of integral equations (on the two smaller domains) could then be used to solve for $\Gamma_{1}$ and $u$ on $\Gamma \backslash \Gamma_{0}$. A similar approach was taken in [6] for a different integral equation formulation (using the potential (2.6)-(2.7) instead), supplemented by additional regularization techniques in the iterative algorithms.

Nonlinear system of equations. In our method, we recast the inverse problem of finding $\Gamma_{1}$ from $u_{0}$ as solving a nonlinear system of equations by least-squares, and set up the Gauss-Newton iteration method. Since $u(x)$ depends on the unknown boundary, in the following, we treat $u$ on the entire $\Gamma$ as an independent unknown in addition to the unknown $\Gamma_{1}$, and the inverse problem is cast as a direct problem of finding both $\Gamma_{1}$ and $u$ on $\Gamma$ from the two equations (2.5) and (3.9). The system is most likely inconsistent which means it may not have a unique solution or the solution may not be stable, especially in the presence of noise in the data $u_{0}$. Hence we understand the solution for the system in the nonlinear least-squares sense.

To be more specific, we introduce parametrization of the boundary $\Gamma$ as in (2.23). The dependence of the operators on $\Gamma$ will then be denoted by their dependence on $x$ as $\mathcal{S}=\mathcal{S}(x)$ and $\mathcal{D}=\mathcal{D}(x)$. In fact, after parametrization, the dependence of the integral operators on the unknown boundary is transformed to the dependence of the kernels on the parametrization of the boundary. We can view it obviously in the explicit form of the kernels which are already developed in chapter 2. For convenience, we denote the operators on the left-hand 
side of (2.4) as

$$
\mathcal{F}(x) u \equiv\left(\frac{1}{2} \mathcal{I}+\mathcal{D}(x)\right) u+(\mathcal{S}(x))(p u)
$$

Assume that $x(t)$ is unknown only when $t \in[a, b]$ (i.e. the $\Gamma_{1}$ part), and the rest of the boundary is known and fixed. By treating the composition $u \circ x$ as an independent unknown, we cast the inverse problem as finding $u(t)=(u \circ x)(t)$ for $t \in[0,1]$ and $x(t)$ for $t \in[a, b]$ from the following nonlinear system:

$$
\left\{\begin{aligned}
\mathcal{F}(x) u & =\mathcal{S}(x) g, \\
\mathcal{R}_{0} u & =u_{0} .
\end{aligned}\right.
$$

Note that, with the parametrization, the first equation is an equation for all $t \in[0,1]$, while the second is for $t \in[c, d]$. Moreover, both equations are linear in $u$, and the first equation is nonlinear in $x$ while the second is independent of $x$. We seek a solution to this system as a solution to the nonlinear least-squares problem:

$$
\min _{(u, x)} \frac{1}{2}\|\mathcal{F}(x) u-\mathcal{S}(x) g\|_{L^{2}[0,1]}^{2}+\frac{1}{2}\left\|\mathcal{R}_{0} u-u_{0}\right\|_{L^{2}[c, d]}^{2}
$$

If another set of data $\hat{u}_{0}$ from a different input $\hat{g}$ is also available, we can easily include another set of equations like (3.12) for $\hat{u}$ and consider a system of 4 equations for the 3 unknowns $u, \hat{u}$ and $x$ :

$$
\left\{\begin{aligned}
\mathcal{F}(x) u & =\mathcal{S}(x) g \\
\mathcal{R}_{0} u & =u_{0} \\
\mathcal{F}(x) \hat{u} & =\mathcal{S}(x) \hat{g} \\
\mathcal{R}_{0} \hat{u} & =\hat{u}_{0} .
\end{aligned}\right.
$$

Similar to the situation for one set of data, the solution $(u, \hat{u}, x)$ is found as the minimizer to the least-squares problem for two sets of data:

$$
\begin{gathered}
\min _{(u, \hat{u}, x)} \frac{1}{2}\|\mathcal{F}(x) u-\mathcal{S}(x) g\|_{L^{2}[0,1]}^{2}+\frac{1}{2}\left\|\mathcal{R}_{0} u-u_{0}\right\|_{L^{2}[c, d]}^{2} \\
\quad+\frac{1}{2}\|\mathcal{F}(x) \hat{u}-\mathcal{S}(x) \hat{g}\|_{L^{2}[0,1]}^{2}+\frac{1}{2}\left\|\mathcal{R}_{0} \hat{u}-\hat{u}_{0}\right\|_{L^{2}[c, d]}^{2}
\end{gathered}
$$

When available, multiple sets of data can be used in this fashion to result in a larger system of equations with the common unknown $x$. Specifically, when there are $K$ sets of data from $K$ different inputs, there will be $2 K$ equations for $K+1$ unknowns in the system, and we understand the solution in the nonlinear least-squares sense. 
Gauss-Newton iteration method. We will employ the Gauss-Newton method for the nonlinear least-squares problem (3.13). To this end, we need to find the linearization of the system of equations in (3.12). Since both equations are linear in $u$ and the second one is independent of $x$, we only need to linearize the first equation in (3.12) with respect to $x$. Let the Fréchet derivatives of operators $\mathcal{S}(x)$ and $\mathcal{D}(x)$, with respect to $x$ in the direction of $\xi$, be denoted by $\dot{\mathcal{S}}(x) u \xi$ and $\dot{\mathcal{D}}(x) u \xi$, respectively. Both operators are bilinear in $u$ and $\xi$. Here $\xi$ is assumed to have support in the interval $[a, b]$.

By the Gauss-Newton method for the nonlinear least-squares problem (3.13), we compute the update step $(\mu, \xi)$ from the linearization of (3.12) at the current iterate $(u, x)$ :

$$
\left\{\begin{aligned}
\mathcal{F}(x) \mu+(\dot{\mathcal{D}}(x) u+\dot{\mathcal{S}}(x)(p u-g)) \xi & =-\mathcal{F}(x) u+\mathcal{S}(x) g, \\
\mathcal{R}_{0} \mu & =-\mathcal{R}_{0} u+u_{0} .
\end{aligned}\right.
$$

The new iterate is then set to be $\left(u^{+}, x^{+}\right)=(u+\mu, x+\xi)$. When solving (3.16) in the linear least-squares sense, we add Tikhonov regularization only for $\xi$, using its second derivative with respect to the parameter $t$ :

$$
\min _{(\mu, \xi)} \frac{1}{2}\left\|\mathcal{F}(x) \mu+\mathcal{Q}(u, x) \xi-e_{1}(u, x)\right\|_{L^{2}[0,1]}^{2}+\frac{1}{2}\left\|\mathcal{R}_{0} \mu-e_{2}(u)\right\|_{L^{2}[c, d]}^{2}+\frac{\alpha}{2}\left\|\xi^{\prime \prime}\right\|_{L^{2}[a, b]}^{2},
$$

where $\mathcal{Q}(u, x)=\dot{\mathcal{D}}(x) u+\dot{\mathcal{S}}(x)(p u-g), e_{1}(u, x)$ and $e_{2}(u)$ are the two right-hand sides in (3.16), and $\alpha>0$ is a regularization parameter. If another data set $\hat{u}_{0}$ from another input $\hat{g}$ is available, then an additional set of equations like (3.16) can be included to form a larger system of 4 equations for the 3 updates $(\mu, \hat{\mu}, \xi)$.

We further introduce a finite-dimensional approximation space in which we seek $\xi(t)$ :

$$
\xi(t) \in \operatorname{span}\left\{\xi^{(1)}(t), \xi^{(2)}(t), \cdots, \xi^{(m)}(t)\right\}
$$

where the basis functions $\xi^{(j)}(t)$ are pre-selected, and have $C_{0}^{2}[a, b]$ components. In the implementation we use cubic $B$-splines as the components of these basis functions. Hence $\xi$ is sought as the form

$$
\xi(t)=\sum_{j=1}^{m} q_{j} \xi^{(j)}(t)
$$

and the unknown boundary $\Gamma_{1}$ is thus represented by the coefficients $\left\{q_{j}\right\}_{j=1}^{m}$. With this 
assumption on $\xi$, the system (3.16) becomes a system for $\mu(\cdot)$ and $\left\{q_{j}\right\}_{j=1}^{m}$ :

$$
\left\{\begin{aligned}
\mathcal{F}(x) \mu+\sum_{j=1}^{m} q_{j}(\dot{\mathcal{D}}(x) u+\dot{\mathcal{S}}(x)(p u-g)) \xi^{(j)} & =-\mathcal{F}(x) u+\mathcal{S}(x) g, \\
\mathcal{R}_{0} \mu & =-\mathcal{R}_{0} u+u_{0},
\end{aligned}\right.
$$

or in the simplified form:

$$
\left\{\begin{aligned}
\mathcal{F}(x) \mu+\tilde{\mathcal{Q}} q & =-\mathcal{F}(x) u+\mathcal{S}(x) g, \\
\mathcal{R}_{0} \mu & =-\mathcal{R}_{0} u+u_{0} .
\end{aligned}\right.
$$

where $\tilde{\mathcal{Q}}$ consists of the column vectors $\mathcal{Q} \xi^{(j)}, j=1,2, \cdots, m$.

Explicit form of the Fréchet derivatives of the integral operators. From the explicit form (2.24)-(2.25) of the integral operators $\mathcal{S}(x)$ and $\mathcal{D}(x)$ after the parametrization, we can also express explicitly their Fréchet derivatives by formally computing the derivatives of the kernels.

The Fréchet derivative of $\mathcal{S}(x)$ in the direction of $\xi$ is computed by:

$$
(\dot{\mathcal{S}}(x) u \xi)(t)=\lim _{h \rightarrow 0} \frac{(\mathcal{S}(x+h \xi) u)(t)-(\mathcal{S}(x) u)(t)}{h},
$$

and similarly, we can find $\dot{\mathcal{D}}(x) u \xi$. They can be expressed as:

$$
(\dot{\mathcal{S}}(x) u \xi)(t)=\int_{0}^{1} \dot{A}(t, s) u(s) d s \quad \text { and } \quad(\dot{\mathcal{D}}(x) u \xi)(t)=\int_{0}^{1} \dot{B}(t, s) u(s) d s
$$

where

with

$$
\dot{A}(t, s)=\frac{1}{2 \pi}\left\{-A_{1}(t, s)+\frac{x^{\prime}(s) \cdot \xi^{\prime}(s)}{\left|x^{\prime}(s)\right|} \ln \frac{1}{|x(t)-x(s)|}\right\}
$$

$$
A_{1}(t, s)= \begin{cases}\frac{(x(t)-x(s)) \cdot(\xi(t)-\xi(s))}{|x(t)-x(s)|^{2}}\left|x^{\prime}(s)\right|, & t \neq s, \\ \frac{x^{\prime}(t) \cdot \xi^{\prime}(t)}{\left|x^{\prime}(t)\right|}, & t=s\end{cases}
$$

and

$$
\dot{B}(t, s)=\frac{1}{2 \pi}\left\{\begin{aligned}
& \frac{\xi^{\prime}(s)^{\perp} \cdot(x(t)-x(s))+x^{\prime}(s)^{\perp} \cdot(\xi(t)-\xi(s))}{|x(t)-x(s)|^{2}} \\
&-\frac{2\left[x^{\prime}(s)^{\perp} \cdot(x(t)-x(s))\right][(x(t)-x(s)) \cdot(\xi(t)-\xi(s))]}{|x(t)-x(s)|^{4}}, \quad t \neq s, \\
& \frac{\xi^{\prime}(t)^{\perp} \cdot x^{\prime \prime}(t)+x^{\prime}(t)^{\perp} \cdot \xi^{\prime \prime}(t)}{2\left|x^{\prime}(t)\right|^{2}}-\frac{\left[x^{\prime}(t)^{\perp} \cdot x^{\prime \prime}(t)\right]\left[x^{\prime}(t) \cdot \xi^{\prime}(t)\right]}{\left|x^{\prime}(t)\right|^{4}}, t=s
\end{aligned}\right.
$$


Note that $\dot{A}$ has a logarithmic singularity, while $\dot{B}$ is continuous. These kernels are all linear in $\xi$. The expressions for these kernels are needed to set up the numerical calculation of solutions for the relevant integral equations that resulted from the Gauss-Newton steps (3.16) or (3.19).

\subsubsection{Numerical Implementation and Examples}

In this section, we present an implementation of our Gauss-Newton iterative methods for the recovery of the unknown Robin boundary $\Gamma_{1}$ from measurements on $\Gamma_{0}$, as well as examples of some recovery results.

Domain setup. The overall shape of the domain for our numerical examples is a rounded rectangle with $1: 5$ aspect ratio; the unknown part $\Gamma_{1}$ is on the top side while the part $\Gamma_{0}$ for measurements is on the bottom, as shown in Figure 3.4 below. The parameter ranges for $\Gamma_{1}$ and $\Gamma_{0}$ are $[a, b]=[0.1,0.4]$ and $[c, d]=[0.6,0.9]$, respectively. The Robin coefficient $p$ is set to be 10 on $\Gamma_{1}$ and 0 elsewhere on $\Gamma$. We choose two particular inputs $g$ and $\hat{g}$ as

$$
g(t)=\left\{\begin{array}{ll}
1 & \text { if } t \in[0.45,0.55] \\
0 & \text { elsewhere }
\end{array} \quad \text { and } \quad \hat{g}(t)= \begin{cases}1 & \text { if } t \in[0,0.05] \cup[0.95,1] \\
0 & \text { elsewhere }\end{cases}\right.
$$

Note that these choices are independent of the unknown Robin boundary $\Gamma_{1}$, and we have chosen inputs whose supports do not overlap with $\Gamma_{0}$.

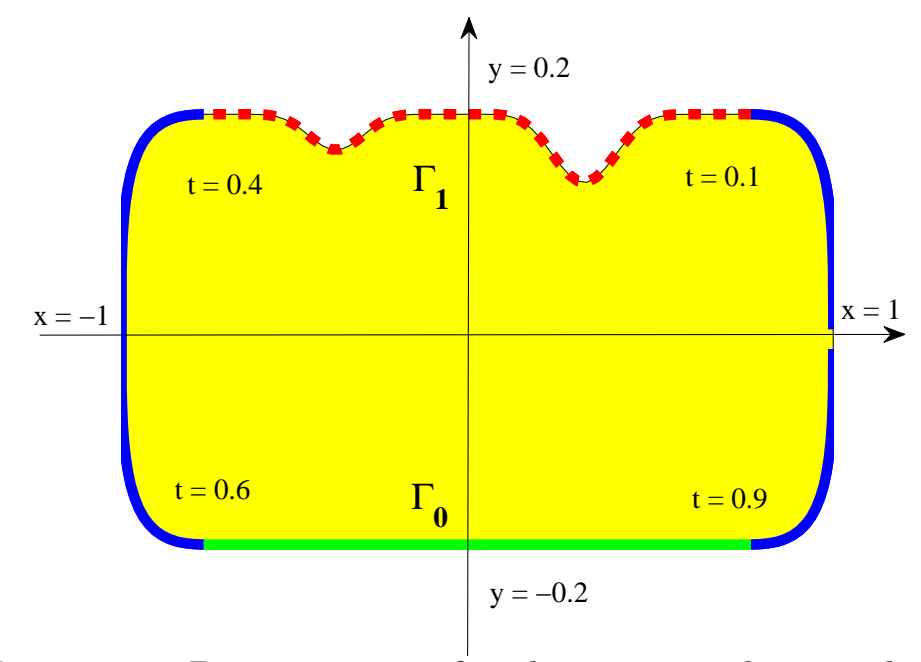

Figure 3.4: Domain setup for the numerical examples. 
Discretization. The interval $[0,1]$ is divided into $n$ equal-length intervals with nodes $\left\{t_{i}\right\}_{i=0}^{n}\left(t_{n}\right.$ identified with $\left.t_{0}\right)$ for our numerical experiments. Since the integral kernels involved have at most a logarithmic singularity, we employ Nyström's method with trigonometric interpolation on regular grids (see e.g. [21]) for the discretization of the integral operators. As for the basis functions for $\xi$ in (3.18), we choose $m$ cubic $B$-splines $B_{j}(t)$ with equally-spaced knots, supported in $[0.1,0.4]$, and set $\xi^{(j)}(t)=\left(0, B_{j}(t)\right)$. We are able to use the exact derivatives for $x(t)$ and $\xi(t)$ that are needed in the integral kernels.

For a true profile $\Gamma$, the synthetic data $u_{0}(t)\left(u\right.$ on $\left.\Gamma_{0}\right)$ corresponding to an input $g$ are simulated by solving the potential $\varphi$ in (2.7) and using (2.6) for $u$. To obtain data with noise, we add random noise of a given level (relative to the $L^{2}$-norm of the data) to the simulated data as

$$
u_{0}^{(\text {noise })}(t)=u_{0}(t)+\delta\left\|u_{0}(t)\right\|_{2} X(t)
$$

where $X(t)$ represents random numbers uniformly distributed on the interval $(-1,1)$. Moreover, each of the true $\Gamma_{1}$ profiles in our test examples is chosen outside the class of curves (3.18) where the iterative algorithm looks for approximate solutions.

After the discretization, the system (3.19) or (3.20), is reduced to the linear system of equations for the unknown vectors $\mu=\left[\mu\left(t_{1}\right), \mu\left(t_{2}\right), \cdots, \mu\left(t_{n}\right)\right]^{T}$ and $q=\left[q_{1}, q_{2}, \cdots, q_{m}\right]^{T}$ :

$$
\left[\begin{array}{ll}
F & Q \\
R_{0} & O
\end{array}\right]\left[\begin{array}{l}
\mu \\
q
\end{array}\right]=\left[\begin{array}{l}
e_{1} \\
e_{2}
\end{array}\right]
$$

where the matrices $F, Q$ and $R_{0}$ are the discretization of the operators $\mathcal{F}, \tilde{\mathcal{Q}}$ and $\mathcal{R}_{0}$ in (3.20), and the vectors $e_{1}, e_{2}$ result from the discretization of the right hand side functions. By the Gauss-Newton method, this system is then solved by the following normal equations, with the addition of a regularization term in $\xi$ as in (3.17):

$$
\left[\begin{array}{cc}
F^{T} F+R_{0}^{T} R_{0} & F^{T} Q \\
Q^{T} F & Q^{T} Q+\alpha D^{T} D
\end{array}\right]\left[\begin{array}{c}
\mu \\
q
\end{array}\right]=\left[\begin{array}{c}
F^{T} e_{1}+R_{0}^{T} e_{2} \\
Q^{T} e_{1}
\end{array}\right]
$$

where the matrix $D^{T} D$ results from the regularization for $\xi^{\prime \prime}$ using (3.18). The particular matrix $F^{T}$ stands for the discretization of the dual of the operator $\mathcal{F}$. Suppose there are $n_{0}$ nodal points in the $t$-range of $\Gamma_{0}$. Then (3.23) has $n+n_{0}$ equations and $n+m$ unknowns, and $(3.24)$ is an $(n+m) \times(n+m)$ system. When two sets of data $u_{0}$ and $\hat{u}_{0}$ from two 
different inputs $g$ and $\hat{g}$ are available, the system (3.23) is expanded into

$$
\left[\begin{array}{ccc}
F & O & Q \\
R_{0} & O & O \\
O & F & \hat{Q} \\
O & R_{0} & O
\end{array}\right]\left[\begin{array}{c}
\mu \\
\hat{\mu} \\
q
\end{array}\right]=\left[\begin{array}{c}
e_{1} \\
e_{2} \\
\hat{e}_{1} \\
\hat{e}_{2}
\end{array}\right],
$$

which is then solved through the normal equations with the addition of the regularization in $\xi$ as

$$
\left[\begin{array}{ccc}
F^{T} F+R_{0}^{T} R_{0} & O & F^{T} Q \\
O & F^{T} F+R_{0}^{T} R_{0} & F^{T} \hat{Q} \\
Q^{T} F & \hat{Q}^{T} F & Q^{T} Q+\hat{Q}^{T} \hat{Q}+\alpha D^{T} D
\end{array}\right]\left[\begin{array}{c}
\mu \\
\hat{\mu} \\
q
\end{array}\right]=\left[\begin{array}{c}
F^{T} e_{1}+R_{0}^{T} e_{2} \\
F^{T} \hat{e}_{1}+R_{0}^{T} \hat{e}_{2} \\
Q^{T} e_{1}+\hat{Q}^{T} \hat{e}_{1}
\end{array}\right]
$$

Note that the matrices $F$ and $F^{T}$ depend only on $\Gamma$ and not on the input $g$, hence the first two diagonal blocks in (3.26) are the same. When more data sets are available, they can be easily incorporated by expanding the systems (3.25)-(3.26) in the obvious way. If there are $K$ sets of data, the resulting systems will have $K n+m$ unknowns, and as $K$ becomes larger, it will be necessary to devise iterative methods in solving the large normal systems, such as Jacobi or Gauss-Seidel, by taking advantage of the favorable matrix structure in systems like (3.26).

Stopping criteria. We start each iteration with the initial $\Gamma_{1}$ as the straight line on the top of the domain (Figure 3.4), and terminate the iteration as soon as the solution to (3.24) or (3.26) satisfies

$$
\frac{1}{m} \sqrt{\sum_{j=1}^{m} q_{j}^{2}} \leq \epsilon
$$

with a predetermined stopping parameter $\epsilon>0$. In all the examples tested, we observe that this quantity is monotonically decreasing throughout nearly every iteration, for both cases of noise-free and noisy data. It should be noted that this observation does not suggest the convergence of the algorithm; in fact, we have also observed that a smaller value of $\epsilon$ does not in general improve the quality of the recovered $\Gamma_{1}$. The selection of this particular quantity as the stopping criterion can be viewed as an additional regularization treatment for the reconstruction algorithm to counter the ill-posedness of the problem. 
Numerical examples. In the numerical examples, we set $n=200, m=27$, and $\epsilon=10^{-5}$. We choose three true profiles of $\Gamma_{1}$ with different characteristics to test our recovery algorithm, which are shown in the figures below as the red dashed lines. These profiles cannot be attained exactly by our algorithm even if no noise is added to the simulated data, since they are not from the same class where the algorithm looks for solutions. Our reconstructions are the blue solid lines. The choice of the regularization parameter $\alpha$ is by experiment using a number of convenient numerical values and by visual inspection, which are by no means the "best" possible choices in any quantitative sense. Typically a larger $\alpha$ is needed for the case of noisy data.

Example 3.5 Numerical results of noise-free data, using one set and two sets of data.

In Figure 3.5 we present the recovery results (blue solid lines) using noise-free data, from one set of data $u_{0}$ for input $g$ (left column), and two sets of data $u_{0}$ and $\hat{u}_{0}$ for inputs $g$ and $\hat{g}$ (right column). These data are simulated by the indirect formulation through (2.6)-(2.7) using the input functions $g$ and $\hat{g}$ from (3.21). For simpler true profiles in the first two examples, one set of data is sufficient for a satisfactory recovery of the Robin boundary $\Gamma_{1}$. On the other hand, it is clear that we can recover the true profile of $\Gamma_{1}$ better with two sets of data, especially in the last example where the profile is slightly more detailed. Note that, even though there is no noise added to the simulated data in these examples, the true profiles cannot be attained exactly by the algorithm.

Example 3.6 Numerical results of 1\%-noisy data, using one set and two sets of data.

In this example, we test the algorithm using data that are contaminated with random noise. As expected, the problem is very ill-posed, and the addition of the random noise in the simulated data indeed makes it difficult for the algorithm to recover the Robin boundary well. In fact, in our experiments, the recovery result from the algorithm depends on the particular realization of the random variable $X$ in (3.22). To better illustrate the effect of random noise in the data on the recovery results, for each example in Figure 3.6, we present in the same plot the results from 10 sets of data with 10 realizations of the random variable $X$; that is, each solid curve represents the recovery result from the data generated by (3.22) with one particular realization of the uniformly distributed random variable $X$ (an $n_{0}$-vector). The relative level of random noise added in $(3.22)$ is set to $1 \%(\delta=0.01)$. In 

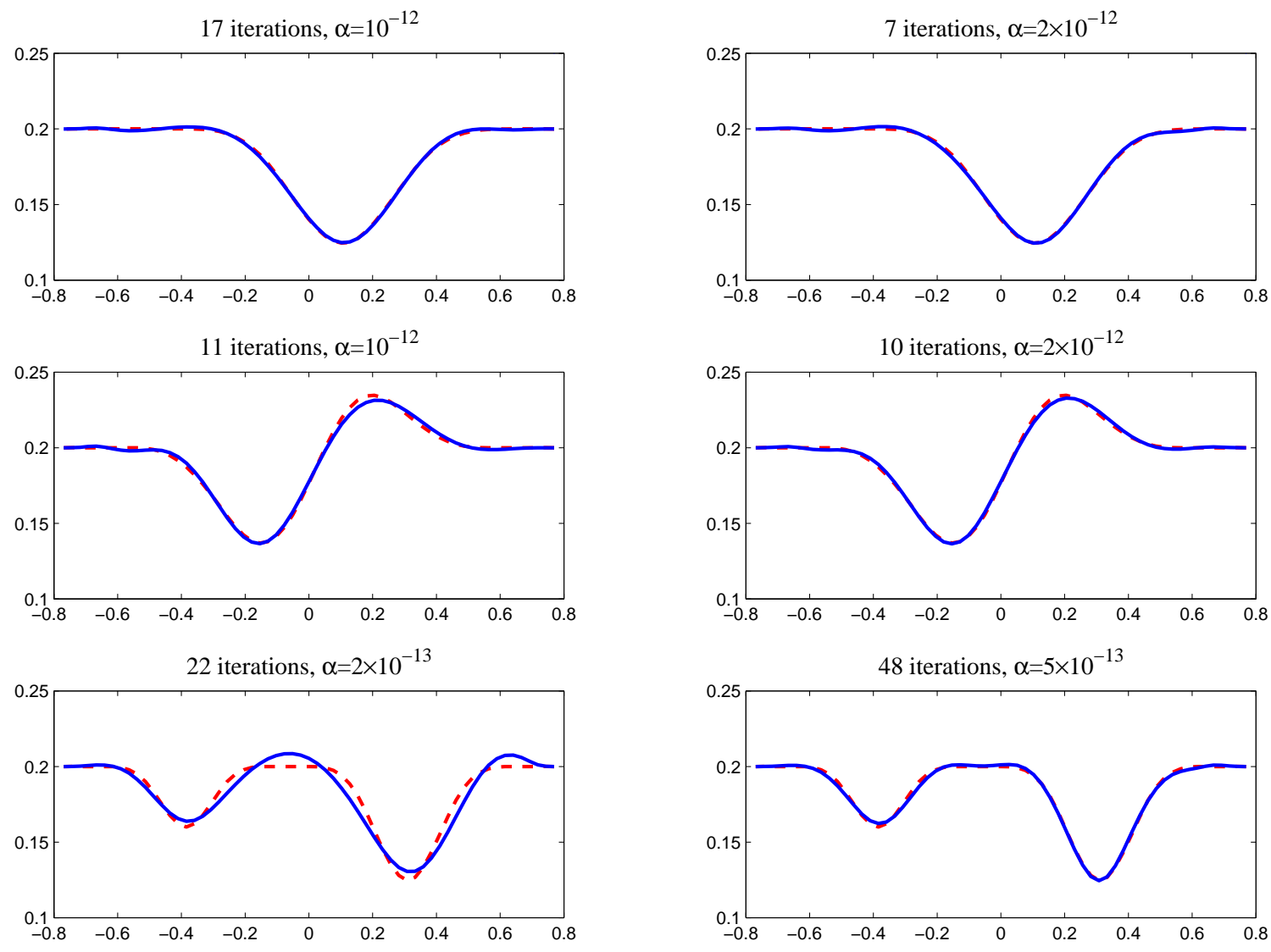

Figure 3.5: Recovery of $\Gamma_{1}$ with noise-free data: from one set of data $u_{0}$ corresponding to input $g$ (left) and from two sets of data $u_{0}$ and $\hat{u}_{0}$ corresponding to inputs $g$ and $\hat{g}$ (right).

the case only one data set $u_{0}^{\text {(noise) }}$ is available, the effect of the random noise in data on the recovery results is noticeable (the left column in Figure 3.6), especially on the right half of $\Gamma_{1}$, perhaps because of the particular choice of the input pattern $g$ whose support is on the left vertical side of $\Gamma$ (see (3.21) and Figure 3.4). On the other hand, the results are much more satisfactory when we have two sets of data $u_{0}^{\text {(noise) }}$ and $\hat{u}_{0}^{\text {(noise) }}$ available for use in the recovery algorithm (the right column in Figure 3.6).

Remarks. We conclude with a few remarks on the problem in general and on our numerical methods in particular.

(i) The aspect ratio of the domain in this inverse problem is a key factor for the ill- 

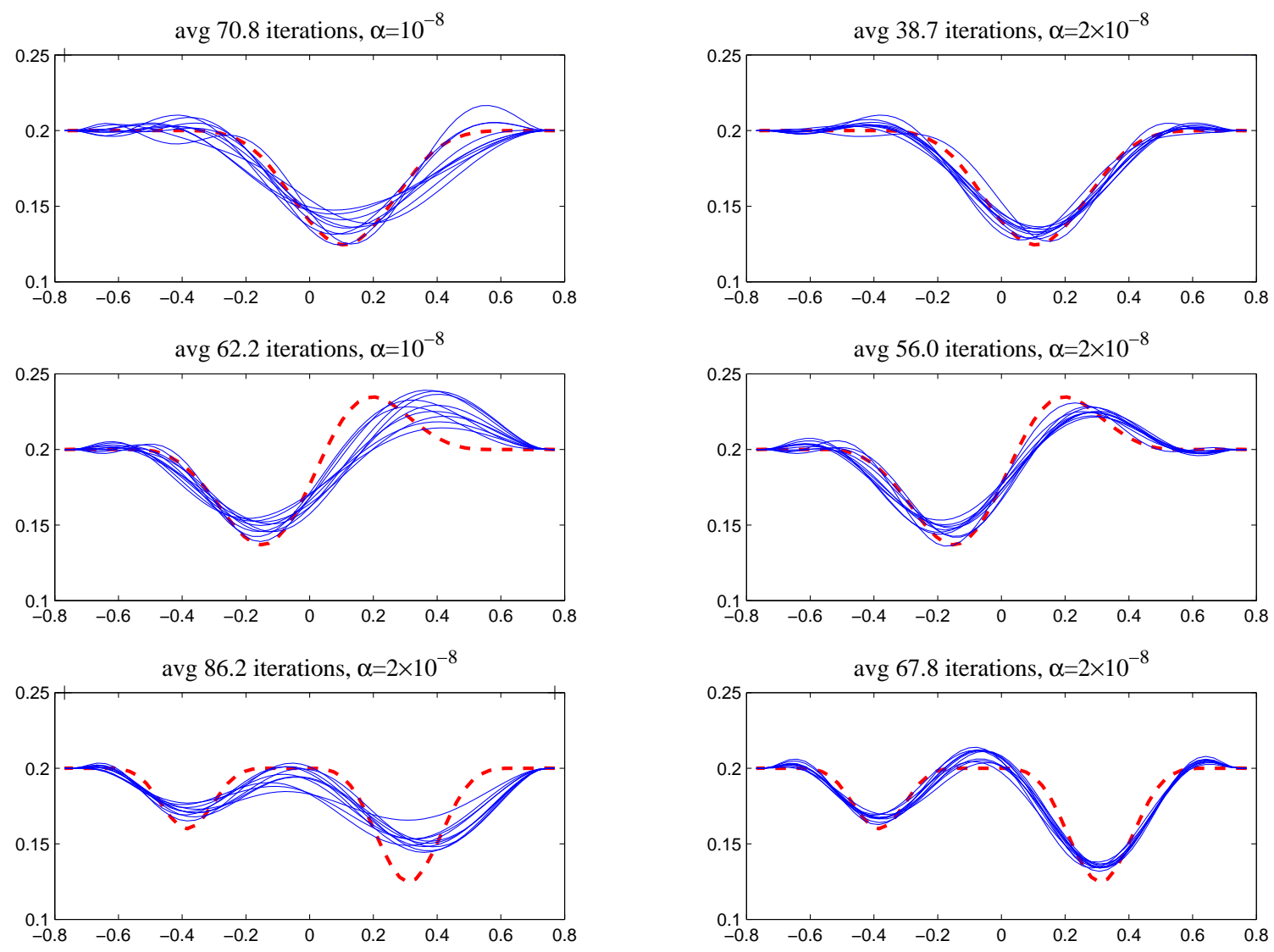

Figure 3.6: Recovery of $\Gamma_{1}$ with $1 \%$-noisy data: from one set of data $u_{0}^{\text {(noise) }}$ corresponding to input $g$ (left) and from two sets of data $u_{0}^{\text {(noise) }}$ and $\hat{u}_{0}^{\text {(noise) }}$ corresponding to inputs $g$ and $\hat{g}$ (right).

posedness of the problem, when everything else is kept relatively the same. This problem is similar to the Cauchy problem on $\Gamma_{0}$ for the Laplacian, whose ill-posedness is well known, and it is more ill-posed when $\Gamma_{1}$ is farther away from $\Gamma_{0}$. In the studies by $[17,14]$ using the PDE model (1.1), it is assumed that the domain is thin, and numerical examples use 1:10 (ours is 1:5) as the aspect ratio. We too observed in our experiments that our algorithms would work better for cases of smaller aspect ratio (i.e. $\Gamma_{1}$ is closer to $\Gamma_{0}$ ), more so with noisy data. The numerical method given by [14] also assumes that the unknown profile be a small perturbation from a known profile, while we do not make such assumption for our GaussNewton method. Other factors, such as the relative position and size of $\Gamma_{0}$ and $\Gamma_{1}$ on the 
domain boundary, the relative magnitude of the Robin coefficient $p$, and the pattern/profile of the input function $g$ (see below), also affect the severity of the ill-posedness of the problem.

(ii) The input functions are often chosen as a characteristic function of an interval in $t$. In [14], the support of such $g$ is chosen to coincide with $\Gamma_{0}$, and in our examples in Figure 3.5 and Figure 3.6, we only use input functions whose supports are disjoint from $\Gamma_{0}$ (see (3.21)). Our experiments seem to indicate that, among the input functions of the same pattern, the location of its support makes some noticeable difference in the quality of the reconstructed $\Gamma_{1}$ by our algorithms. To demonstrate such difference, we present our recovery results in Figure 3.7 using data from the input function

$$
\tilde{g}(t)= \begin{cases}1 & \text { if } t \in[0.7,0.8] \\ 0 & \text { elsewhere }\end{cases}
$$

which has the support within $\Gamma_{0}$ and directly below $\Gamma_{1}$, in contrast to $g$ and $\hat{g}$ from (3.21) whose supports are on the vertical sides of the rounded rectangle. These two plots are to be compared to results using $g$ in the two lower left plots of Figure 3.5 and Figure 3.6. Clearly data corresponding to $\tilde{g}$ yield better reconstruction results than that from $g$, possibly because of the specific configuration of the relative positions of the supports to $\Gamma_{1}$ and $\Gamma_{0}$. Especially in the case of noise-free data, the result using just one set of data from $\tilde{g}$ (Figure 3.7 left) is nearly as good as the result using two data sets from $g$ and $\hat{g}$ (Figure 3.5, bottom right). In situations where the pattern of the input functions can be chosen without restriction, it would be interesting and practical to find out what kinds of input functions, or collection of such if multiple measurements are possible, would give rise to data that will yield better results in recovering $\Gamma_{1}$.

(iii) For solving the system (3.12), an alternative to our Gauss-Newton method (3.16) is to utilize the fact that (3.12) is linear in $u$ and set up the following iteration: Given $x$, solve the linear system

$$
\left\{\begin{aligned}
\mathcal{F}(x) u & =\mathcal{S}(x) g, \\
\mathcal{R}_{0} u & =u_{0}
\end{aligned}\right.
$$

for $u$ by least-squares, then with this $u$, find the Newton direction $\xi$ to update $x$ by the linearized first equation of (3.12)

$$
(\dot{\mathcal{D}}(x) u+\dot{\mathcal{S}}(x)(p u-g)) \xi=-\mathcal{F}(x) u+\mathcal{S}(x) g,
$$



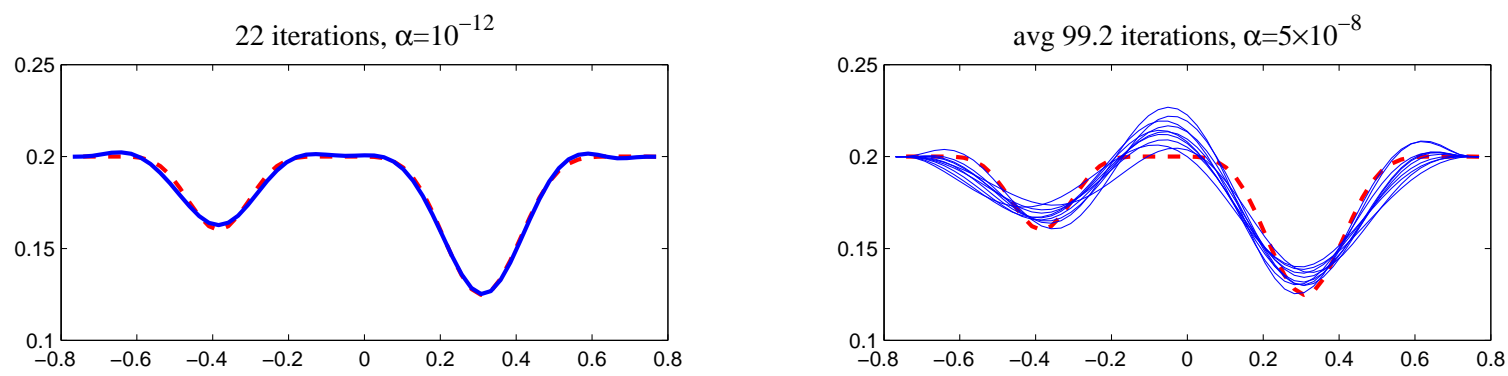

Figure 3.7: Recovery of $\Gamma_{1}$ from one set of data corresponding to input $\tilde{g}$ in (3.28): with noise-free data $\tilde{u}_{0}$ (left) and with $1 \%$-noisy data $\tilde{u}_{0}^{\text {(noise) }}$ (right).

with the addition of a Tikhonov regularization. This would be in the same spirit as the approach by [6] for their formulation. Compared to our Gauss-Newton method, this alternative neglects the $\xi$-term in (3.16) when solving for the $u$-component, hence does not solve the two components simultaneously in a larger coupled system. One advantage is that the systems involved are smaller, but our experiments indicate that this approach does not perform as well as the full Gauss-Newton method for us. We should recognize the importance of setting up the problem this way using the full Gauss-Newton method, since it gives the necessary basis for the design of other iterative methods when needed. In the case when the full system (3.16) or (3.24) is too large, this setup also provides a structure that naturally suggests iterative methods for efficient solutions. Furthermore, as we have shown, this setting can deal effectively and efficiently with the case when multiple data sets are available.

(iv) As seen in other studies, the problem is so ill-posed that, besides the standard Tikhonov-type regularization, it is almost necessary to introduce additional regularization treatments in order for an algorithm to be reasonably successful. In both [6] and our study here, the use of the finite dimensional approximation (3.18) for the unknown $\Gamma_{1}$ is one such treatment, in which the presence of regularization is stronger when the number of basis functions $m$ is smaller. In [6], the number $m$ is chosen adaptively, increasing with iteration; in addition, the update steps in the iteration are scaled so as to include the extra regularization effect. With $m$ fixed, the simple stopping criterion (3.27) we design for the Gauss-Newton method seems to work well in all of our examples; it is practical, and indeed it adds some needed regularization effect in our algorithms to prevent the iteration to continue into an undesirable regime due to the ill-posedness of the problem. 


\section{Chapter 4}

\section{Recovery of the Interface in the Inverse Linear Source Problem}

In this chapter, we study the inverse problem of the recovery of the unknown interface $\partial S$ from a boundary measurement of the solution $U=u_{0}$ on $\Gamma_{0} \subset \partial \Omega$. This problem is known as the inverse linear source problem, and we continue to adopt the integral equation approach to study this inverse problem.

\subsection{Solution by Least Squares}

Based on the integral equations formulation (2.22) as introduced in Chapter 2, we look for an approximate solution $\partial S$ in the least squares sense from the outer partial boundary measurement $u=u_{0}$ on $\Gamma_{0} \subset \partial \Omega$.

We introduce the parametrization of $\partial \Omega$ and $\partial S$ as follows:

$$
\partial \Omega=\left\{z_{0}(t): t \in[0,1]\right\} \quad \text { and } \quad \partial S=\{z(t): t \in[0,1]\}
$$

and denote

$$
u(t)=u\left(z_{0}(t)\right), \quad v(t)=v(z(t)), \quad w(t)=w(z(t)) .
$$

For a numerical implementation reason, we assume that $z(t)$ has the star-like form:

$$
z(t)=r(t)(\cos 2 \pi t, \sin 2 \pi t) \quad \text { for } t \in[0,1],
$$

where $r(t)$ is a periodic function. 
Let $\Gamma_{0}=\left\{z_{0}(t): t \in[a, b]\right\}, 0 \leq a<b \leq 1$. Denote the restriction operator from $\partial \Omega$ to $\Gamma_{0}$ by $\mathcal{R}_{0}$; that is, for $u$ defined on $\partial \Omega, \mathcal{R}_{0} u$ is defined on $\Gamma_{0}$ with $\left(\mathcal{R}_{0} u\right)(x)=u(x)$ for $x \in \Gamma_{0}$. Then, for a given input $g$ on $\partial \Omega$, the measurement $u_{0}$ of $u$ on $\Gamma_{0}$ can be expressed as

$$
\mathcal{R}_{0} u=u_{0} .
$$

A straightforward approach to discover the unknown interior boundary $\partial S$ using the measurement $u_{0}$ on part of the outside boundary $\Gamma_{0}$ is to investigate the dependence of the solution $u(t)$ on $z(t)$, and then find $z(t)$ that produces the "best" match with the data. That is, we find $z(t)$ of $\partial S$ from the nonlinear least-squares problem:

$$
\min _{z(t)} \frac{1}{2}\left\|\mathcal{R}_{0} u(z(t))-u_{0}(t)\right\|_{L^{2}[a, b]}^{2},
$$

which in turn will be solved by a Gauss-Newton iteration. Let $\dot{u}(z) \xi$ be the Fréchet derivative of $u$ with respect to $z$ in the direction of $\xi$. Then we find the Gauss-Newton step $\xi$ at the current iterate $z$ from the linearization of (4.1):

$$
\mathcal{R}_{0} \dot{u}(z) \xi+\mathcal{R}_{0} u(z)-u_{0}(t)=0 .
$$

With our proper assumption of $z(t)$, we seek $\xi$ with the form:

$$
\xi(t)=\rho(t)(\cos 2 \pi t, \sin 2 \pi t) \quad \text { for } t \in[0,1],
$$

where $\rho(t)$ is a periodic function. We find the solution of (4.3) as the solution of the linear least-squares problem, with an additional Tikhonov regularization term for the new radius function $r(t)+\rho(t)$, using its second derivative:

$$
\min _{\xi} \frac{1}{2}\left\|\mathcal{R}_{0} \dot{u}(z) \xi+\mathcal{R}_{0} u(z)-u_{0}(t)\right\|_{L^{2}[a, b]}^{2}+\frac{\alpha}{2}\left\|r^{\prime \prime}(t)+\rho^{\prime \prime}(t)\right\|_{L^{2}[0,1]}^{2} .
$$

Further, we seek $\rho(t)$ in a finite dimensional space spanned by $m$ pre-determined periodic basis functions $\left\{\rho_{j}(t)\right\}_{j=1}^{m}$ :

$$
\rho(t)=\sum_{j=1}^{m} q_{j} \rho_{j}(t) \quad \text { or equivalently } \quad \xi(t)=\sum_{j=1}^{m} q_{j} \xi_{j}(t)
$$

with $\xi_{j}(t)=\rho_{j}(t)(\cos 2 \pi t, \sin 2 \pi t)$. Thus, $\xi$ is represented by the unknown coefficients $\left\{q_{j}(t)\right\}_{j=1}^{m}$, and solving equation (4.3) for $\xi$ becomes solving for the unknown coefficients $\left\{q_{j}(t)\right\}_{j=1}^{m}$ :

$$
\sum_{j=1}^{m} q_{j} \mathcal{R}_{0} \dot{u}_{j}(z)=u_{0}(t)-\mathcal{R}_{0} u(z)
$$


where $\dot{u}_{j}(z)=\dot{u}(z) \xi_{j}$.

To compute the derivatives $\dot{u}_{j}(z)$, we rely on system (2.22) and the Fréthet derivatives of the operators in the system. Let $\dot{\mathcal{S}}_{01} \xi$ be the Fréthet derivative of the operator $\mathcal{S}_{01}$ with respect to $z$ in the direction $\xi$. Similar notations are used for the other operators. From system (2.22), by a formal calculus of variation procedure, we find that the derivatives $(\dot{u}, \dot{v}, \dot{w})$ of $(u, v, w)$ with respect to $z$ in the direction of $\xi$ satisfy

$$
\left[\begin{array}{ccc}
\frac{1}{2} \mathcal{I}_{0}+\mathcal{D}_{0} & -\mathcal{D}_{01} & \mathcal{S}_{01} \\
\mathcal{D}_{10} & \frac{1}{2} \mathcal{I}_{1}-\mathcal{D}_{1} & \mathcal{S}_{1} \\
\mathcal{O}_{10} & \frac{1}{2} \mathcal{I}_{1}+\mathcal{D}_{p} & -\mathcal{S}_{p}
\end{array}\right]\left[\begin{array}{c}
\dot{u} \\
\dot{v} \\
\dot{w}
\end{array}\right]=\left[\begin{array}{c}
\left(\dot{\mathcal{D}}_{01} \xi\right) v-\left(\dot{\mathcal{S}}_{01} \xi\right) w \\
\left(\dot{\mathcal{S}}_{10} \xi\right) g-\left(\dot{\mathcal{D}}_{10} \xi\right) u+\left(\dot{\mathcal{D}}_{1} \xi\right) v-\left(\dot{\mathcal{S}}_{1} \xi\right) w \\
-\left(\dot{\mathcal{D}}_{p} \xi\right) v+\left(\dot{\mathcal{S}}_{p} \xi\right) w
\end{array}\right] .
$$

Then, in particular, the derivative $\dot{u}_{j}(z)$ can be obtained by solving (4.8) with $\xi$ replaced by each corresponding $\xi_{j}=\rho_{j}(t)(\cos (2 \pi t), \sin (2 \pi t)), j=1, \cdots, m$. To see this more specifically, from (4.8), we have:

$$
\left[\begin{array}{ccc}
\frac{1}{2} \mathcal{I}_{0}+\mathcal{D}_{0} & -\mathcal{D}_{01} & \mathcal{S}_{01} \\
\mathcal{D}_{10} & \frac{1}{2} \mathcal{I}_{1}-\mathcal{D}_{1} & \mathcal{S}_{1} \\
\mathcal{O}_{10} & \frac{1}{2} \mathcal{I}_{1}+\mathcal{D}_{p} & -\mathcal{S}_{p}
\end{array}\right]\left[\begin{array}{c}
\dot{u}_{j} \\
\dot{v}_{j} \\
\dot{w}_{j}
\end{array}\right]=\left[\begin{array}{c}
\left(\dot{\mathcal{D}}_{01} \xi_{j}\right) v-\left(\dot{\mathcal{S}}_{01} \xi_{j}\right) w \\
\left(\dot{\mathcal{S}}_{10} \xi_{j}\right) g-\left(\dot{\mathcal{D}}_{10} \xi_{j}\right) u+\left(\dot{\mathcal{D}}_{1} \xi_{j}\right) v-\left(\dot{\mathcal{S}}_{1} \xi_{j}\right) w \\
-\left(\dot{\mathcal{D}}_{p} \xi_{j}\right) v+\left(\dot{\mathcal{S}}_{p} \xi_{j}\right) w
\end{array}\right]
$$

or in the abbreviated form:

$$
\mathcal{F}(z)\left[\begin{array}{c}
\dot{u}_{j} \\
\dot{v}_{j} \\
\dot{w}_{j}
\end{array}\right]=e_{j}\left(\xi_{j}, u, v, w\right)
$$

and we find $\dot{u}_{j}$ as the first component of the solution $\left[\dot{u}_{j}, \dot{v}_{j}, \dot{w}_{j}\right]^{T}$. Thus, with the derivatives $\dot{u}_{j}(z)$ computed, equation (4.7) can be further expressed as solving the unknown coefficient vector $q=\left[q_{1}, q_{2}, \cdots, q_{m}\right]^{T}$ from the following linear system:

$$
\left[\dot{u}_{1}, \dot{u}_{2}, \cdots, \dot{u}_{m}\right]\left[\begin{array}{c}
q_{1} \\
q_{2} \\
\vdots \\
q_{m}
\end{array}\right]=u_{0}-\mathcal{R}_{0} u .
$$

Initial guess. To proceed with a Gauss-Newton iteration, an initial guess is usually needed. The above framework also provides a quick way of finding an initial guess from a 
particular class of simple curves, for example, circles. The family of circles can be obviously parameterized by

$$
z(t)=(a+r \cos 2 \pi t, b+r \sin 2 \pi t)
$$

with parameters $a, b, r$. We can have a Gauss-Newton iteration:

$$
(\hat{a}, \hat{b}, \hat{r})=(a, b, r)+\lambda(\delta a, \delta b, \delta r)
$$

where we have included a scalar $0<\lambda \leq 1$ to ensure that the circle $\hat{z}(\hat{a}, \hat{b}, \hat{r}, t)$ remains within $\Omega$. The direction $(\delta a, \delta b, \delta r)$ is found exactly like the above framework if we identify

$$
\left(q_{1}, q_{2}, q_{3}\right)=(a, b, r) \quad \text { and } \quad \xi_{1}=(1,0), \quad \xi_{2}=(0,1), \quad \xi_{3}=(\cos 2 \pi t, \sin 2 \pi t)
$$

in the above. This turns out to be an effective way of obtaining a good initial guess for our algorithms.

\subsection{The Explicit Forms of the Integral Operators and Their Fréchet Derivatives}

Suppose $\Gamma$ is a smooth simple closed curve in $R^{2}$, and it has the following parametrization

$$
z(t)=\left(x_{1}(t), x_{2}(t)\right) \quad \text { for } t \in[0,1]
$$

where $x_{1}(t)$ and $x_{2}(t)$ are $C^{2}$ periodic functions in $R$ with period 1 . The outward unit normal to the boundary of $\Gamma$ is denoted by $\nu_{z}(t)$, i.e.,

$$
\nu_{z}(t)=\left(x_{2}^{\prime}(t),-x_{1}^{\prime}(t)\right) /\left|z^{\prime}(t)\right|=z^{\prime}(t)^{\perp} /\left|z^{\prime}(t)\right|
$$

where $z^{\prime}(t)^{\perp}=\left(x_{2}^{\prime}(t),-x_{1}^{\prime}(t)\right)$.

Let $F(s)$ be a smooth function on $(0, \infty)$ and have at most a logarithmic singularity at $s=0$. Consider the integral operator

$$
\int_{\Gamma} F(|x-y|) \phi(y) d s_{y}
$$

as a model of the operators we will encounter in (2.22). Then, for each $x=\left(x_{1}, x_{2}\right)$ and $y=z(\tau) \in \Gamma$, we have the following parametric representations:

$$
\begin{aligned}
\int_{\Gamma} F(|x-y|) \phi(y) d s_{y} & =\int_{0}^{1} F(|x-z(\tau)|) \phi(z(\tau))\left|z^{\prime}(\tau)\right| d \tau \\
& \equiv \int_{0}^{1} A(x, z(\tau)) \phi(z(\tau)) d \tau
\end{aligned}
$$




$$
\begin{aligned}
\int_{\Gamma} \frac{\partial F(|x-y|)}{\partial \nu_{y}} \phi(y) d s_{y} & =\int_{0}^{1} F^{\prime}(|x-z(\tau)|) \frac{(z(\tau)-x) \cdot \nu_{z}(\tau)}{|x-z(\tau)|} \phi(z(\tau))\left|z^{\prime}(\tau)\right| d \tau \\
& =\int_{0}^{1} F^{\prime}(|x-z(\tau)|) \frac{(z(\tau)-x) \cdot z^{\prime}(\tau)^{\perp}}{|x-z(\tau)|} \phi(z(\tau)) d \tau \\
& \equiv \int_{0}^{1} B(x, z(\tau)) \phi(z(\tau)) d \tau
\end{aligned}
$$

and

$$
\begin{aligned}
\int_{\Gamma} \frac{\partial F(|x-y|)}{\partial \nu_{x}} \phi(y) d s_{y} & =\int_{0}^{1} F^{\prime}(|x-z(\tau)|) \frac{(x-z(\tau)) \cdot \nu_{x}}{|x-z(\tau)|} \phi(z(\tau))\left|z^{\prime}(\tau)\right| d \tau \\
& \equiv \int_{0}^{1} C(x, z(\tau)) \phi(z(\tau)) d \tau
\end{aligned}
$$

The kernels $B$ and $C$ are related by

$$
C(\hat{z}(t), z(\tau))=B(z(\tau), \hat{z}(t)) \frac{\left|z^{\prime}(\tau)\right|}{\left|\hat{z}^{\prime}(t)\right|}
$$

for any smooth $z$ and $\hat{z}$.

For the fundamental solution $\Phi$ in $(2.2), F(s)=-\frac{1}{2 \pi} \ln s$ and $F^{\prime}(s)=-\frac{1}{2 \pi s}$, and hence the corresponding operators have the kernels as

$$
\begin{aligned}
& A_{\Phi}(x, z(\tau))=-\frac{\left|z^{\prime}(\tau)\right|}{2 \pi} \ln |x-z(\tau)|, \\
& B_{\Phi}(x, z(\tau))= \begin{cases}-\frac{1}{2 \pi} \frac{(z(\tau)-x) \cdot z^{\prime}(\tau)^{\perp}}{|x-z(\tau)|^{2}}, & x \notin \Gamma, \\
\frac{1}{4 \pi} \frac{z^{\prime \prime}(\tau) \cdot z^{\prime}(\tau)^{\perp}}{\left|z^{\prime}(\tau)\right|^{2}}, & x \in \Gamma .\end{cases}
\end{aligned}
$$

Note that, $B_{\Phi}$ is a continuous kernel; while $A_{\Phi}$ has a logarithmic singularity in $\tau$ when $x \in \Gamma$. Note that these are the same kernels we have in (2.24)-(2.25).

For the fundamental solution $\Psi$ in (2.19),

$$
F(s)=\frac{1}{2 \pi} K_{0}\left(q_{0} s\right)=-\frac{1}{2 \pi}\left(J_{0}\left(i q_{0} s\right) \ln s+F_{0}\left(q_{0} s\right)\right)
$$

and

$$
F^{\prime}(s)=\frac{q_{0}}{2 \pi} K_{0}^{\prime}\left(q_{0} s\right)=-\frac{q_{0}}{2 \pi} K_{1}\left(q_{0} s\right)=-\frac{F_{1}\left(q_{0} s\right)}{2 \pi s}
$$

where we set

$$
F_{0}(z)=-K_{0}(z)-J_{0}(i z) \ln \frac{z}{q_{0}}, \quad K_{1}(z)=-K_{0}^{\prime}(z) \quad \text { and } \quad F_{1}(z)=z K_{1}(z) .
$$


Here $J_{0}$ is the Bessel function of order 0 of the first kind. From the asymptotic expansions of $J_{0}, K_{0}$ and $K_{1}$ (see Appendix), we see that $F_{0}(z)$ and $F_{1}(z)$ are both smooth for $z>0$ and

$$
\begin{aligned}
& F_{0}(z) \sim \gamma_{0}+\ln \frac{q_{0}}{2}+\left(\gamma_{0}+\ln \frac{q_{0}}{2}\right) \frac{z^{2}}{4}+\cdots \quad \text { as } \quad z \rightarrow 0^{+} . \\
& F_{1}(z) \sim 1+\frac{z^{2}}{2} \ln \frac{z}{2}+\cdots
\end{aligned}
$$

The corresponding operators have the kernels as

$$
\begin{aligned}
A_{\Psi}(x, z(\tau)) & =-\frac{\left|z^{\prime}(\tau)\right|}{2 \pi}\left\{J_{0}\left(i q_{0}|x-z(t)|\right) \ln |x-z(t)|+F_{0}\left(q_{0}|x-z(\tau)|\right)\right\} \\
& \equiv A_{\Psi}^{(0)}(x, z(\tau)) \ln |x-z(t)|+A_{\Psi}^{(1)}(x, z(\tau)), \\
B_{\Psi}(x, z(\tau))= & \frac{F_{1}\left(q_{0}|x-z(\tau)|\right)}{2 \pi} \cdot \frac{(x-z(\tau)) \cdot z^{\prime}(\tau)^{\perp}}{|x-z(\tau)|^{2}} .
\end{aligned}
$$

Note that $A_{\Psi}^{(0)}, A_{\Psi}^{(1)}$ and $B_{\Psi}$ are all continuous in $\tau$ for any $x$, and $B_{\Psi}$ has the same diagonal value as $B_{\Phi}$.

With the explicit forms of $A_{\Phi}, B_{\Phi}, A_{\Psi}$ and $B_{\Psi}$, we can express the integral operators involved in system (2.22) explicitly. We adopt the parametrization of $\partial S$ and $\partial \Omega$ developed in the previous section:

$$
\partial \Omega=\left\{z_{0}(t): t \in[0,1]\right\} \quad \text { and } \quad \partial S=\{z(t): t \in[0,1]\} .
$$

For simplicity, set

$$
u(t)=u\left(z_{0}(t)\right), \quad v(t)=v(z(t)), \quad w(t)=w(z(t)),
$$

and the kernels for the single-layer and double-layer potential operators are labeled by $A$ and $B$ respectively, following the same indexing as for the operators. For example,

$$
\left(\mathcal{S}_{01} v\right)(t)=\int_{0}^{1} A_{01}(t, \tau) v(\tau) d \tau \quad \text { with } \quad A_{01}(t, \tau)=A_{\Phi}\left(z_{0}(t), z(\tau)\right)
$$

and so on. Specifically, the kernels of the operators are given by

$$
\begin{array}{llll}
\mathcal{S}_{0}: & A_{0}(t, \tau)=A_{\Phi}\left(z_{0}(t), z_{0}(\tau)\right), & \mathcal{D}_{0}: & B_{0}(t, \tau)=B_{\Phi}\left(z_{0}(t), z_{0}(\tau)\right), \\
\mathcal{S}_{01}: & A_{01}(t, \tau)=A_{\Phi}\left(z_{0}(t), z(\tau)\right), & \mathcal{D}_{01}: & B_{01}(t, \tau)=B_{\Phi}\left(z_{0}(t), z(\tau)\right), \\
\mathcal{S}_{10}: & A_{10}(t, \tau)=A_{\Phi}\left(z(t), z_{0}(\tau)\right), & \mathcal{D}_{10}: & B_{10}(t, \tau)=B_{\Phi}\left(z(t), z_{0}(\tau)\right), \\
\mathcal{S}_{1}: & A_{1}(t, \tau)=A_{\Phi}(z(t), z(\tau)), & \mathcal{D}_{1}: & B_{1}(t, \tau)=B_{\Phi}(z(t), z(\tau)), \\
\mathcal{S}_{p}: & A_{p}(t, \tau)=A_{\Psi}(z(t), z(\tau)), & \mathcal{D}_{p}: & B_{p}(t, \tau)=B_{\Psi}(z(t), z(\tau)) .
\end{array}
$$


For each derivative operator, we can also provide its kernel. For example, for $\dot{\mathcal{S}}_{01}(z)$, the linear operator of $\dot{\mathcal{S}}_{01}(z) \xi$ acting on $w$ has a kernel denoted by $\dot{A}_{01}$ :

$$
\left(\dot{\mathcal{S}}_{01}(z) \xi w\right)(t)=\int_{0}^{1} \dot{A}_{01}(t, \tau) w(\tau) d \tau .
$$

From the formulas of the integral operators developed previously, we can find the explicit form for the derivative operators as:

$$
\begin{aligned}
\dot{A}_{01}(t, \tau)= & -\frac{1}{2 \pi} \frac{z^{\prime}(\tau) \cdot \xi^{\prime}(\tau)}{\left|z^{\prime}(\tau)\right|} \ln \left|z_{0}(t)-z(\tau)\right|+\frac{\left|z^{\prime}(\tau)\right|}{2 \pi} \cdot \frac{\left(z_{0}(t)-z(\tau)\right) \cdot \xi(\tau)}{\left|z_{0}(t)-z(\tau)\right|^{2}}, \\
\dot{A}_{10}(t, \tau)= & -\frac{\left|z_{0}^{\prime}(\tau)\right|}{2 \pi} \cdot \frac{\left(z(t)-z_{0}(\tau)\right) \cdot \xi(t)}{\left|z(t)-z_{0}(\tau)\right|^{2}}, \\
\dot{B}_{01}(t, \tau)= & \frac{1}{2 \pi} \frac{-\xi(\tau) \cdot z^{\prime}(\tau)^{\perp}+\left(z_{0}(t)-z(\tau)\right) \cdot \xi^{\prime}(\tau)^{\perp}}{\left|z_{0}(t)-z(\tau)\right|^{2}} \\
& +\frac{1}{\pi} \frac{\left[\left(z_{0}(t)-z(\tau)\right) \cdot z^{\prime}(\tau)^{\perp}\right]\left[\left(z_{0}(t)-z(\tau)\right) \cdot \xi(\tau)\right]}{\left|z_{0}(t)-z(\tau)\right|^{4}}, \\
\dot{B}_{10}(t, \tau)= & \frac{1}{2 \pi} \frac{\xi(t) \cdot z_{0}^{\prime}(\tau)^{\perp}}{\left|z(t)-z_{0}(\tau)\right|^{2}}-\frac{1}{\pi} \frac{\left[\left(z(t)-z_{0}(\tau)\right) \cdot z_{0}^{\prime}(\tau)^{\perp}\right]\left[\left(z(t)-z_{0}(\tau)\right) \cdot \xi(t)\right]}{\left|z(t)-z_{0}(\tau)\right|^{4}}, \\
\dot{A}_{1}(t, \tau)= & -\frac{1}{2 \pi} \frac{z^{\prime}(\tau) \cdot \xi^{\prime}(\tau)}{\left|z^{\prime}(\tau)\right|} \ln |z(t)-z(\tau)|-\frac{\left|z^{\prime}(\tau)\right|}{2 \pi} \cdot \frac{(z(t)-z(\tau)) \cdot(\xi(t)-\xi(\tau))}{|z(t)-z(\tau)|^{2}}, \\
\dot{A}_{p}(t, \tau)= & -\frac{J_{0}\left(i q_{0}|z(t)-z(\tau)|\right)}{2 \pi} \cdot \frac{z^{\prime}(\tau) \cdot \xi^{\prime}(\tau)}{\left|z^{\prime}(\tau)\right|} \ln |z(t)-z(\tau)| \\
& -\frac{F_{0}\left(q_{0}|z(t)-z(\tau)|\right)}{2 \pi} \cdot \frac{z^{\prime}(\tau) \cdot \xi^{\prime}(\tau)}{\left|z^{\prime}(\tau)\right|} \\
& -\frac{\left|z^{\prime}(\tau)\right| F_{1}\left(q_{0}|z(t)-z(\tau)|\right)}{2 \pi} \cdot \frac{(z(t)-z(\tau)) \cdot(\xi(t)-\xi(\tau))}{|z(t)-z(\tau)|^{2}},
\end{aligned}
$$




$$
\begin{aligned}
\dot{B}_{1}(t, \tau)= & \frac{1}{2 \pi} \frac{(\xi(t)-\xi(\tau)) \cdot z^{\prime}(\tau)^{\perp}+(z(t)-z(\tau)) \cdot \xi^{\prime}(\tau)^{\perp}}{|z(t)-z(\tau)|^{2}} \\
& -\frac{1}{\pi} \frac{\left[(z(t)-z(\tau)) \cdot z^{\prime}(\tau)^{\perp}\right][(z(t)-z(\tau)) \cdot(\xi(t)-\xi(\tau))]}{|z(t)-z(\tau)|^{4}}, \\
\dot{B}_{p}(t, \tau)= & \frac{F_{1}\left(q_{0}|z(t)-z(\tau)|\right)}{2 \pi} \cdot \frac{(\xi(t)-\xi(\tau)) \cdot z^{\prime}(\tau)^{\perp}+(z(t)-z(\tau)) \cdot \xi^{\prime}(\tau)^{\perp}}{|z(t)-z(\tau)|^{2}} \\
& -\frac{F_{2}\left(q_{0}|z(t)-z(\tau)|\right)}{\pi} \cdot \frac{\left[(z(t)-z(\tau)) \cdot z^{\prime}(\tau)^{\perp}\right][(z(t)-z(\tau)) \cdot(\xi(t)-\xi(\tau))]}{|z(t)-z(\tau)|^{4}}
\end{aligned}
$$

where in $\dot{B}_{p}$ we denote

$$
F_{2}(z)=z K_{1}(z)+\frac{1}{2} z^{2} K_{0}(z) \sim 1-\frac{1}{4} z^{2}+\cdots \quad \text { as } z \rightarrow 0^{+},
$$

and we have used the relation $K_{1}^{\prime}(z)=-K_{0}(z)-\frac{1}{z} K_{1}(z)$ in the calculation of $F_{1}^{\prime}(z)$. The first four kernels are continuous since $z(t) \neq z_{0}(\tau)$ for any $t$ and $\tau$. Each of the kernels $\dot{A}_{1}$ and $\dot{A}_{p}$ has a logarithmic singular part and a continuous part, and the continuous parts have diagonal values as, respectively,

$$
-\frac{1}{2 \pi} \frac{z^{\prime}(\tau) \cdot \xi^{\prime}(\tau)}{\left|z^{\prime}(\tau)\right|} \text { and } \quad-\frac{1+\gamma_{0}+\ln \frac{q_{0}}{2}}{2 \pi} \cdot \frac{z^{\prime}(\tau) \cdot \xi^{\prime}(\tau)}{\left|z^{\prime}(\tau)\right|} .
$$

The kernels $\dot{B}_{1}$ and $\dot{B}_{p}$ are continuous, with the same diagonal value given by

$$
\dot{B}_{1}(\tau, \tau)=\dot{B}_{p}(\tau, \tau)=\frac{1}{4 \pi} \frac{\xi^{\prime \prime}(\tau) \cdot z^{\prime}(\tau)^{\perp}+z^{\prime \prime}(\tau) \cdot \xi^{\prime}(\tau)^{\perp}}{\left|z^{\prime}(\tau)\right|^{2}}-\frac{1}{2 \pi} \frac{\left[z^{\prime \prime}(\tau) \cdot z^{\prime}(\tau)^{\perp}\right]\left[z^{\prime}(\tau) \cdot \xi^{\prime}(\tau)\right]}{\left|z^{\prime}(\tau)\right|^{4}} .
$$

These expressions for the integral operators and their Fréchet derivatives are needed in computing numerical solutions to the system of integral equations (2.22) and in solving the Gauss-Newton steps in the iterations.

\subsection{Numerical Implementation and Examples}

In this section, we present the implementation details of our straightforward method to recover the unknown $\partial S$ from the partial boundary measurement $u_{0}$ on $\partial \Omega$, as well as reconstruction results in numerical examples. 
For the sake of simplicity, we assume the measurement $u_{0}$ is given on the whole $\partial \Omega$; that is, $\left(\mathcal{R}_{0} u\right)(x(t))=u(t)$ for $t \in[0,1]$ on $\partial \Omega$. Consider the linear least-squares problem (4.5):

$$
\min _{\xi} \frac{1}{2}\left\|\dot{u}(z) \xi+u(z)-u_{0}(t)\right\|_{L^{2}[0,1]}^{2}+\frac{\alpha}{2}\left\|r^{\prime \prime}(t)+\rho^{\prime \prime}(t)\right\|_{L^{2}[0,1]}^{2},
$$

where $\xi=\rho(t)(\cos (2 \pi t), \sin (2 \pi t))$. With the representation for $\rho$ :

$$
\rho(t)=\sum_{j=1}^{m} q_{j} \rho_{j}(t)
$$

the above minimization problem becomes:

$\min _{q} \frac{1}{2}\left\|\dot{u}(z) \sum_{j=1}^{m} q_{j} \rho_{j}(t)(\cos (2 \pi t), \sin (2 \pi t))+u(z)-u_{0}(t)\right\|_{L^{2}[0,1]}^{2}+\frac{\alpha}{2}\left\|r^{\prime \prime}(t)+\sum_{j=1}^{m} q_{j} \rho_{j}^{\prime \prime}(t)\right\|_{L^{2}[0,1]}^{2}$

where $q=\left[\begin{array}{lll}q_{1} & \cdots & q_{m}\end{array}\right]^{T}$ is the unknown coefficient vector. The notation can be further simplified as:

$$
\min _{q} \frac{1}{2}\left\|\sum_{j=1}^{m} q_{j} \dot{u}_{j}(z)+u(z)-u_{0}(t)\right\|_{L^{2}[0,1]}^{2}+\frac{\alpha}{2}\left\|r^{\prime \prime}(t)+\sum_{j=1}^{m} \rho_{j}^{\prime \prime}(t)\right\|_{L^{2}[0,1]}^{2}
$$

where $\dot{u}_{j}(z)=\dot{u}(z) \rho_{j}(t)(\cos (2 \pi t), \sin (2 \pi t))$. Let

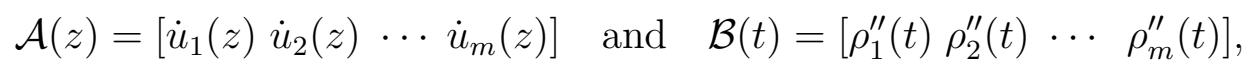

then the above least-squares problem can be expressed in the system form as:

$$
\min _{q} \frac{1}{2}\left\|\mathcal{A} q+u(z)-u_{0}(t)\right\|_{L^{2}[0,1]}^{2}+\frac{\alpha}{2}\left\|r^{\prime \prime}(t)+\mathcal{B} q\right\|_{L^{2}[0,1]}^{2} .
$$

Suppose, after discretization, $\mathcal{A}$ and $\mathcal{B}$ are represented by matrices $A$ and $B$, respectively. Then the discretized normal equation becomes

$$
\left(A^{T} A+\alpha B^{T} B\right) q=A^{T}\left(u_{0}-u\right)-\alpha B^{T} r^{\prime \prime}
$$

Note. When the measurement $u_{0}$ is known only on part of the boundary $\partial \Omega$, i.e., $u_{0}$ has support on $\Gamma_{0} \subset \partial \Omega$, we can still follow the same structure of system (4.15) and its normal equation (4.16) to find the solution. All we need to do is restrict the function $u$ on the support $\Gamma_{0}$ of $u_{0}$. If multiple sets of data are available on the outer boundary, we suggest one approach to incorporate the additional information into our system by expanding the leastsquares problem (4.15) and its corresponding normal equation (4.16) as follows. Suppose 
there are two sets of data $u_{0}$ and $\hat{u}_{0}$ from two different inputs $g$ and $\hat{g}$, respectively. Then we find our solution from the least-squares problem:

$$
\min _{q} \frac{1}{2}\left\|\mathcal{A} q+u-u_{0}\right\|_{L^{2}[0,1]}^{2}+\frac{1}{2}\left\|\hat{\mathcal{A}} q+\hat{u}-\hat{u}_{0}\right\|_{L^{2}[0,1]}^{2}+\frac{\alpha}{2}\left\|r^{\prime \prime}+\mathcal{B} q\right\|_{L^{2}[0,1]}^{2} .
$$

The corresponding normal equation is given as

$$
\left(A^{T} A+\hat{A}^{T} \hat{A}+\alpha B^{T} B\right) q=A^{T}\left(u_{0}-u\right)+\hat{A}^{T}\left(u_{0}-\hat{u}\right)-\alpha B^{T} r^{\prime \prime} .
$$

Note that, if $A$ is a $n \times m$ matrix, then the resulting system of the normal equation for multiple sets of data is still $m \times m$, the same size as in the case of a single data set.

Numerical results. Now we illustrate the feasibility of our method by presenting numerical results. For simplicity, we take the outer boundary $\partial \Omega$ as a circle centered at the origin and with radius 2 ; the measurement $u_{0}$ is given on $\Gamma_{0} \subset \partial \Omega$, as shown in Figure 4.1. We choose two particular inputs $g$ and $\hat{g}$ as the characteristic functions: $g(t)=1$ for $t \in[0,1]$ and $\hat{g}=1$ for $t \in[0,0.5]$ and 0 elsewhere.

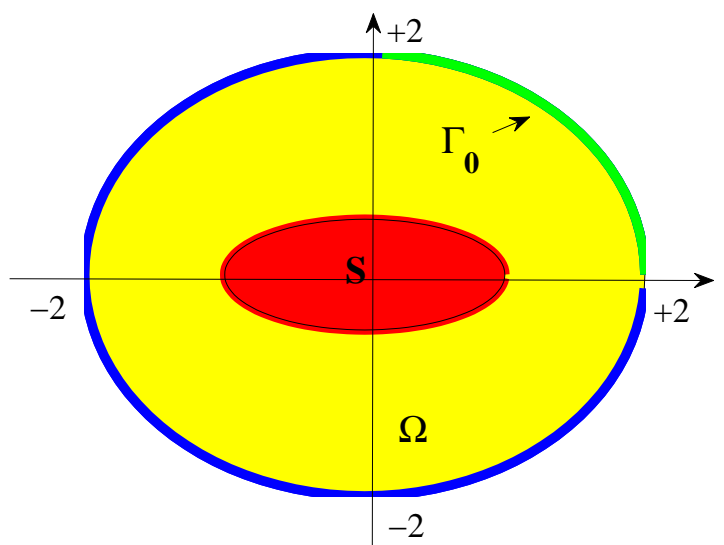

Figure 4.1: Domain setup for the numerical examples.

For discretization, we divide $[0,1]$ into $n$ equidistant subintervals with quadrature points $t_{i}=i / n, i=0, \cdots, 1\left(t_{n}=t_{0}\right)$. Since the integral kernels involved have at most a logarithmic singularity, we employ the Nyström's method with trigonometric interpolation to deal with the singularities for the discretization of the integral operators. As for the basis functions of $\rho(t)$, we choose $\rho_{j}(t)$ as $m C^{1}$-periodic cubic $B$-splines with equally-spaced knots and support in $[0,1]$. This guarantees the computation of the exact derivatives of $z(t)$ and $\xi(t)$ that are needed in the integral kernels. 
The synthetic data $u_{0}$, measurement of $u(t)$ on $\Gamma_{0}$, is simulated by solving (2.22) for $u(t)$ from a given true profile of $\partial S$ and a prescribed input function $g(t)$. To obtain data with noise, we add to the simulated data $u_{0}(t)$ random noise with a given noisy level $\delta$ relative to the $L^{2}$-norm of the data:

$$
u_{0}^{(n o i s e)(t)}=u_{0}(t)+\delta\left\|u_{0}(t)\right\|_{2} X(t)
$$

where $X(t)$ represents random numbers uniformly distributed on the interval $(-1,1)$. Further, all of the true profiles of $\partial S$ chosen in our examples do not belong to the class of curves (4.6) where our algorithm looks for solutions.

We start each iteration from our initial guess, a circle within $\Omega$ chosen by our initial guess algorithm. As stated before, the initial guess algorithm follows the same idea as our straightforward method, that is, to look for a circle within $\Omega$ that minimize the $L^{2}$ norm of the equation $u(t)-u_{0}(t)$. The initial guess provides the Gauss-Newton iteration a relatively closed location where to look for solutions. We terminate the iteration when the solution to (4.16) satisfies

$$
\sqrt{\sum_{j=1}^{m} q_{j}^{2}} \leq \epsilon
$$

with a predetermined stopping parameter $\epsilon>0$. This can be considered as an additional regularization treatment to the ill-posedness of the problem in our reconstructions. From our observation, in the examples we test below, the stopping quantity is decreasing throughout nearly each iteration for both cases of noise free and noisy data. However, we should note that this observation does not suggest the convergence of the algorithm, since smaller $\epsilon$ does not in general improve the reconstruction quality of the unknown boundary $\partial S$.

In the numerical examples, we set $n=100, m=25$, and $\epsilon=10^{-5}$. We choose different true profiles of $\partial S$ to test our recovery algorithm. In the figures, the true profiles are shown as the red dashed lines and the constructions are represented by the blue solid lines. The regularization parameter $\alpha$ is chosen by experiment and inspection. In general, a larger $\alpha$ is needed for a heavier noisy data.

Example 4.1 Recovery of different true profiles of $\partial S$ using noise-free data.

In Figure 4.2, we recover 3 true profiles of $\partial S$ from noise-free data that are given on the entire outer boundary $\partial \Omega$. As can be seen, the reconstructions are almost exact for all the profiles except at the area where indentation occurs. 
7 iterations, $\alpha=1 \times 10^{-12}$

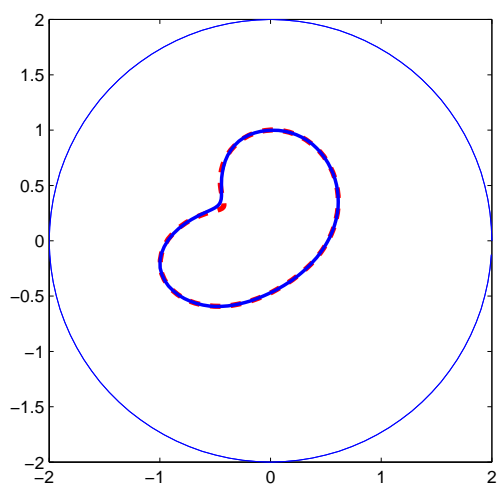

Bean shape
8 iterations, $\alpha=1 \times 10^{-10}$

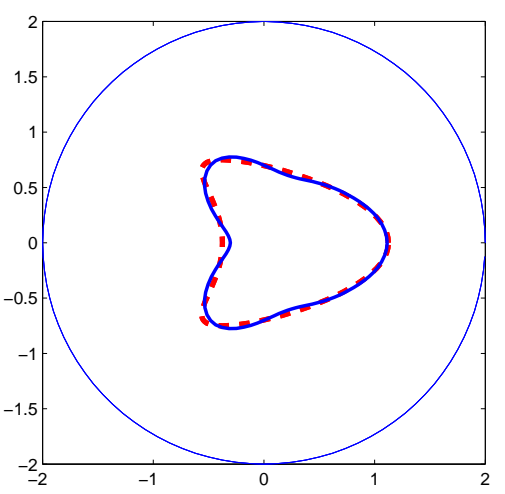

Kite shape
7 iterations, $\alpha=1 \times 10^{-12}$

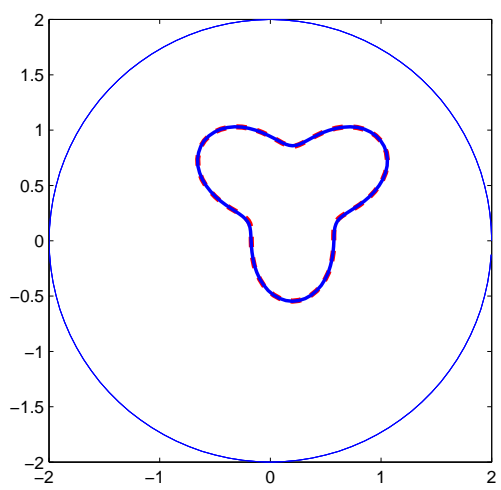

Rose shape

(Measurement $u_{0}(t)$ is given on the entire outer circle.)

Figure 4.2: Recovery of different true profiles of $\partial S$ with noise-free data.

Example 4.2 Recovery of different true profiles of $\partial S$ using 1\%-noisy data.

Next, we test our algorithm using noisy data. Indeed, our recovery becomes more difficult when the data are contaminated by random noise. In Figure 4.3, to better illustrate the effect of random noise on the recovery results, we present in each recovery example the results from 5 sets of data with 5 corresponding realizations of random noise. The approximations are much less satisfactory especially in the concave part of the true profiles of $\partial S$, however, our algorithm can still determine the rough shape and location of the true profiles.

Example 4.3 Recovery of the interface $\partial S$ from measurement on part of outer circle.

In this example, we illustrate the recovery results for the bean shape using 1\%-noisy data and the data are given only on part of $\partial \Omega$. Figure 4.4 shows 3 reconstructions for the data that are known on an entire circle, on half a circle, and on a quarter circle, respectively. As expected, with less information given on the outer boundary, the more difficulty we have in recovering the true profile of the interior interface. In the case when $u_{0}$ is known only on a quarter circle(the last example), we can barely obtain the 'bean' shape of the true profile but a "circle" body, possibly due to the initial guess configuration.

Example 4.4 Recovery of the interface $\partial S$ using one set and two sets of noisy data. 


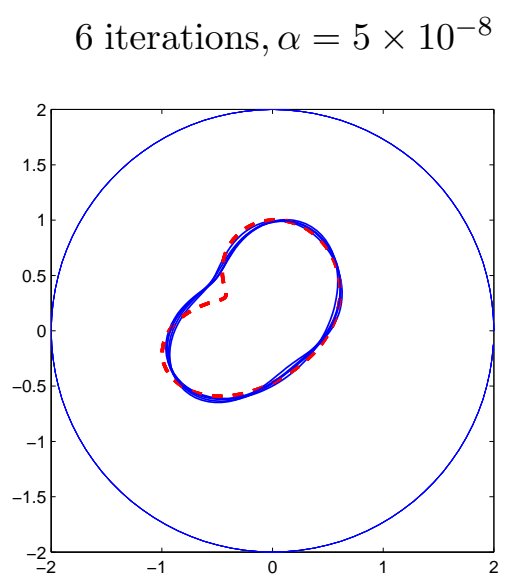

Bean shape
6 iterations, $\alpha=5 \times 10^{-8}$

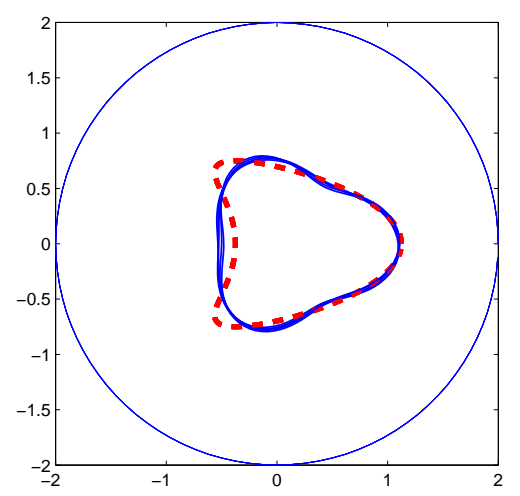

Kite shape
7 iterations, $\alpha=3 \times 10^{-8}$

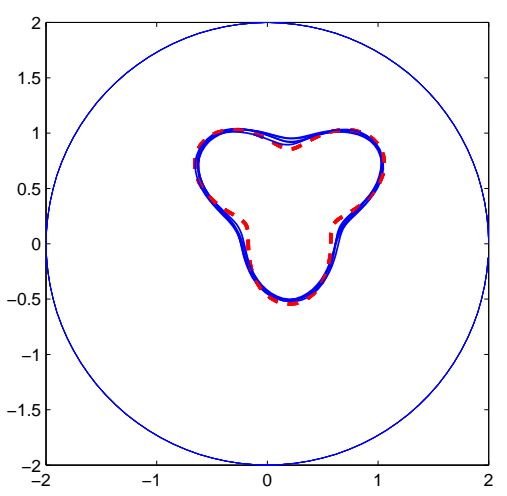

Rose shape

(Measurement $u_{0}(t)$ is given on the entire outer circle.)

Figure 4.3: Recovery of different true profiles of $\partial S$ with 1\%-noisy data.

For our last example, we compare the recovery results with one set of data and the results with two sets of data, using boundary measurement with added noise. We employ the approach suggested in (4.17)-(4.18) for using two sets of data. Figure 4.5 shows the recoveries using one set of data for input $g$ (left) and two sets of data for inputs $g$ and $\hat{g}$ (right) from the boundary measurements $u_{0}$ and $\hat{u}_{0}$ on the entire outer circle. By comparing the results, we do not observe significant improvements in the result using more data sets. We also tested for the noise-free case and for the case where $u_{0}$ is given only on the upper-half circle, and did not observe much difference between the use of one set or two sets of data.

Remark. Our least-squares approach based on the integral equations formulation is a straightforward method for the inverse linear source problem. The system involved has only one equation and it has the same parametric range as the partial outer boundary measurement $u_{0}$. Compared to other PDE methods, this approach has much less computational cost; once we set up the forward solver with system (2.22), the derivatives $\dot{u}$ in the iterative system (4.7) can be easily computed. As a by-product, this framework provides a quick method of obtaining a very good initial guess for the Newton-type iterative schemes. When the measurement $u_{0}$ is known on the whole outer boundary $\Omega$, our reconstruction algorithm for the unknown $\partial S$ works very well for the noise-free case. Results are less satisfactory for the noisy data, but still quite accurate in terms of location and overall shapes. When the 
6 iterations, $\alpha=5 \times 10^{-8}$

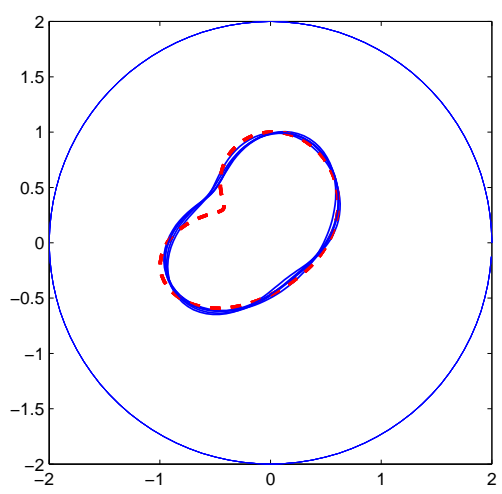

$u_{0}(t)$ on the entire outer circle

$$
t \in[0,1]
$$

6 iterations, $\alpha=5 \times 10^{-8}$

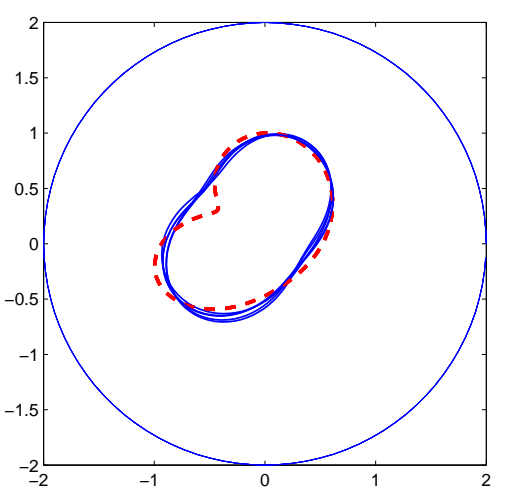

$u_{0}(t)$ on the upper half circle

$$
t \in[0,0.5]
$$

4.2 iterations, $\alpha=7 \times 10^{-8}$

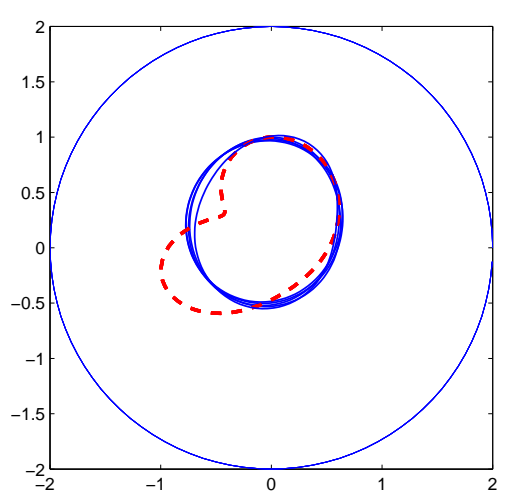

$u_{0}(t)$ on the first quarter circle

$$
t \in[0,0.25]
$$

Figure 4.4: Recovery of $\partial S$ with $1 \%$-noisy data on partial $\partial \Omega$.

measurement is given only on part of the outer boundary, the recovery becomes very difficult and indeed, the inverse problem is highly ill-posed. The analysis of the ill-posedness in this situation is studied by [15] from the view point of "recovery from limited angle data". If there are multiple sets of data available on the outer partial boundary, our method provides a simple basic structure to incorporate the extra information into the system, but we have not observed significant improvement in the reconstruction results. 
6.2 iterations, $\alpha=5 \times 10^{-8}$

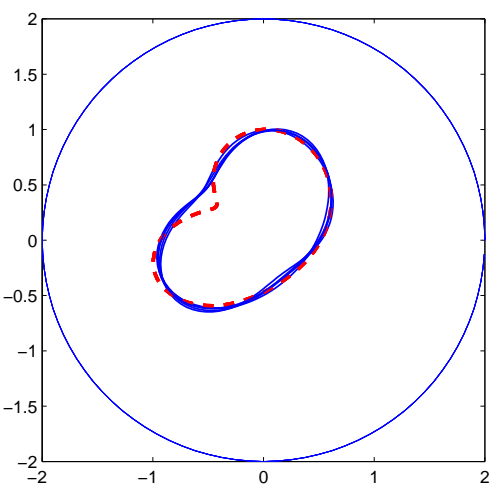

from one data set $u_{0}(t)$
6.4 iterations, $\alpha=2 \times 10^{-7}$

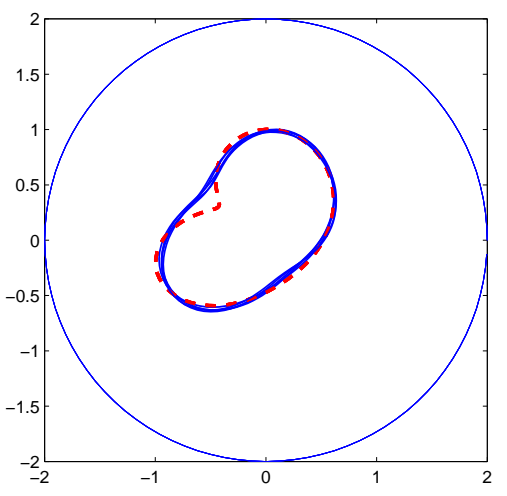

from two data sets $u_{0}(t)$ and $\hat{u}_{0}(t)$

Figure 4.5: Recovery of $\partial S$ using one set versus two sets of $1 \%$ noisy data. 


\section{Appendix A}

\section{The Modified Bessel Function $K_{0}$}

Here we state the asymptotic behavior for the modified Bessel function $K_{0}(z)$ of order 0 of the second kind near the singularity $z=0$. We need these properties in the formulations of the linear source problem since the fundamental solution $\Psi$ is given by $K_{0}$. More complete details can be found in [2].

For each integer $n$, let $J_{n}(z)$ and $Y_{n}(z)$ denote the Bessel functions of order $n$ of the first and second kind, respectively. They are linear independent solutions to the Bessel's equation

$$
z^{2} y^{\prime \prime}(z)+z y^{\prime}(z)+\left(z^{2}-n^{2}\right) y(z)=0 .
$$

The modified Bessel functions $K_{n}$ are closely related to $J_{n}$ and $Y_{n}$.

We are interested in the 0 order modified Bessel function $K_{0}$ :

$$
K_{0}(z)=\frac{\pi}{2} i\left(J_{0}(i z)+i Y_{0}(i z)\right) .
$$

Using the series expressions of $J_{0}$ and $Y_{0}$,

$$
\begin{gathered}
J_{0}(i z)=\sum_{k=0}^{\infty} \frac{(-1)^{k}}{(k !)^{2}}\left(\frac{i z}{2}\right)^{2 k}=\sum_{k=0}^{\infty} \frac{1}{(k !)^{2}}\left(\frac{z}{2}\right)^{2 k}, \\
Y_{0}(i z)=\frac{2}{\pi}\left(\ln \frac{i z}{2}+\gamma_{0}\right) J_{0}(i z)-\frac{2}{\pi} \sum_{k=1}^{\infty} \frac{(-1)^{k} a_{k}}{(k !)^{2}}\left(\frac{i z}{2}\right)^{2 k} \\
=i J_{0}(i z)+\frac{2}{\pi}\left(\ln \frac{z}{2}+\gamma_{0}\right) J_{0}(i z)-\frac{2}{\pi} \sum_{k=1}^{\infty} \frac{a_{k}}{(k !)^{2}}\left(\frac{z}{2}\right)^{2 k},
\end{gathered}
$$


we deduce that

$$
\begin{aligned}
K_{0}(z) & =-\left(\ln \frac{z}{2}+\gamma_{0}\right) J_{0}(i z)+\sum_{k=1}^{\infty} \frac{a_{k}}{(k !)^{2}}\left(\frac{z}{2}\right)^{2 k} \\
& =-\left(\ln \frac{z}{2}+\gamma_{0}\right) \sum_{k=0}^{\infty} \frac{1}{(k !)^{2}}\left(\frac{z}{2}\right)^{2 k}+\sum_{k=1}^{\infty} \frac{a_{k}}{(k !)^{2}}\left(\frac{z}{2}\right)^{2 k} .
\end{aligned}
$$

Here $a_{k}=\sum_{m=1}^{k} \frac{1}{m}$, and $\gamma_{0}=\lim _{k \rightarrow \infty}\left(a_{k}-\ln k\right)=0.5772 \ldots$ is the Euler constant.

We will also need the derivative of $K_{0}$ :

$$
K_{0}^{\prime}(z)=-K_{1}(z)=\frac{\pi}{2}\left(J_{1}(i z)+i Y_{1}(i z)\right)
$$

where

$$
\begin{gathered}
J_{1}(i z)=\sum_{k=0}^{\infty} \frac{(-1)^{k}}{k !(k+1) !}\left(\frac{i z}{2}\right)^{2 k+1}=i \sum_{k=0}^{\infty} \frac{1}{k !(k+1) !}\left(\frac{z}{2}\right)^{2 k+1}, \\
Y_{1}(i z)=-\frac{2}{\pi i z}+\frac{2}{\pi}\left(\ln \frac{i z}{2}+\gamma_{0}-\frac{1}{2}\right) J_{1}(i z)-\frac{1}{\pi} \sum_{k=1}^{\infty}\left(2 a_{k}-1+\frac{1}{k+1}\right) \frac{(-1)^{k}}{k !(k+1) !}\left(\frac{i z}{2}\right)^{2 k+1} \\
=-\frac{2}{\pi i z}+i J_{1}(i z)+\frac{2}{\pi}\left(\ln \frac{z}{2}+\gamma_{0}-\frac{1}{2}\right) J_{1}(i z)-\frac{i}{\pi} \sum_{k=1}^{\infty} \frac{2 a_{k}-1+\frac{1}{k+1}}{k !(k+1) !}\left(\frac{z}{2}\right)^{2 k+1} .
\end{gathered}
$$

Hence

$$
\begin{aligned}
K_{0}^{\prime}(z) & =-\frac{1}{z}+\left(\ln \frac{z}{2}+\gamma_{0}-\frac{1}{2}\right) i J_{1}(i z)+\frac{1}{2} \sum_{k=1}^{\infty} \frac{2 a_{k}-1+\frac{1}{k+1}}{k !(k+1) !}\left(\frac{z}{2}\right)^{2 k+1} \\
& =-\frac{1}{z}-\left(\ln \frac{z}{2}+\gamma_{0}-\frac{1}{2}\right) \sum_{k=0}^{\infty} \frac{1}{k !(k+1) !}\left(\frac{z}{2}\right)^{2 k+1}+\frac{1}{2} \sum_{k=1}^{\infty} \frac{2 a_{k}-1+\frac{1}{k+1}}{k !(k+1) !}\left(\frac{z}{2}\right)^{2 k+1} .
\end{aligned}
$$

These series expressions for $K_{0}$ and $K_{0}^{\prime}$ describes the asymptotic behavior of these functions near the singularity $z=0$. 


\section{Bibliography}

[1] H. T. Banks, F. Kojima, and W. P. Winfree, Boundary estimation problems arising in thermal tomography, Inverse Problems, 6(1990), pp. 897-922.

[2] W. W. Bell, Special Functions for Scientists and Engineers, Van Nostrand, London, 1968.

[3] K. Bryan and L.F. Caudill, An inverse problem in thermal imaging, SIAM J. Appl. Math., 56(1996), pp. 715-735.

[4] S. Busenberg and W. Fang, Identification of semiconductor contact resistivity, Quart. Appl. Math., 49(1991), pp. 639-649.

[5] S. Chaabane, M. Jaoua, Identification of Robin coefficients by means of boundary measurements, Inverse Problems, (15)1999, pp. 1425-1438.

[6] F. Cakoni, R. Kress, Integral equations for inverse problems in corrosion detection from partial Cauchy data, Inverse problems and imaging, Vol 1, No.2, 2007, pp. 229-245.

[7] D. Colton and R. Kress, Inverse Acoustic and Electromagnetic Scattering Theory, 2nd ed., Springer-Verlag, Berlin, 1998.

[8] W. Fang and E. Cumberbatch, Inverse problems for MOSFET contact resistivity, SIAM J. Appl. Math., 52(1992), pp. 699-709.

[9] W. Fang and K. Ito, Identification of contact regions in semiconductor transistors by level set methods, J. Comput. Appl. Math., 159(2003), pp.399-410.

[10] W. Fang and M. Lu, A fast wavelet collocation method for an inverse boundary value problem, Int. J. Numer. Meth. Engng., 59 (2004), pp. 1563-1585. 
[11] W. Fang and S. Zeng, A direct solution for the Robin inverse problem, J. Integral Eq. Appl., (2009), to appear.

[12] W. Fang and S. Zeng, Numerical recovery of Robin boundary from boundary measurements for the Laplace equation, J. Comput. Appl. Math., 224(2009), pp.573-580.

[13] D. Fasini and G. Inglese, An inverse problem for Laplace's equation: theoretical results and numerical methods, Inverse Problems, (15)1999, pp. 41-48.

[14] D. Fasino, G. Inglese, and F. Mariani, Corrosion detection in conducting boundaries: II, Linearization, stability and discretization, Inverse Problem, 23(2007), pp. 1101-1114.

[15] F. Hettlich and W. Rundell, Recovery of the support of a source term in an elliptic differential equation, Inverse Problems, 13(1997), pp. 959-976.

[16] G. Inglese, An inverse problem in corrosion detection, Inverse Problems, 13 (1997), pp. 977-994.

[17] G. Inglese and F. Mariani, Corrosion detection in conducting boundaries, Inverse Problem, 20(2004), pp. 1207-1215.

[18] V. Isakov, Inverse Problems for Partial Differential Equations, 2nd ed., Springer, New York, 2006.

[19] P. Kaup and F. Santosa, Nondestructive evaluation of corrosion damage using electrostatic measurements, J. Nondestructive Eval., (14)1995, pp. 127-136.

[20] A. Kirsch, An Introduction to the Mathematical Theory of Inverse Problems, SpringerVerlag, New York, 1996.

[21] R. Kress, Linear Integral Equations, 2nd ed., Springer-Verlag, New York, 1999.

[22] R. Kress and W. Rundell, Nonlinear integral equations and the iterative solution for an inverse boundary value problem, Inverse Problems, (21)2005, pp. 1207-1223.

[23] O. A. Ladyzhenskaya and N. N. Ural'tzeva, Linear and Quasilinear Elliptic Equations, Academic Press, London, UK, 1968. 
[24] F. Lin and W. Fang, A linear integral equation approach to the Robin inverse problem, Inverse Problems, 21(2005), pp. 1757-1772.

[25] W. H. Loh, Modeling and measurement of contact resistances, Stanford Electronics Labs, Technical Report No.G830-1, December 1987.

[26] W. H. Loh, K. Saraswat, and R. W. Dutton, Analysis and scaling of Kelvin resistors for extraction of specific contact Problems, IEEE Electron Device Letters, (6)1985, pp. 105-108.

[27] M. Lu, An inverse boundary value problem from semiconductor modeling, PhD Dissertation, 2003, West Virginia University.

[28] W. McLean, Strongly Elliptic Systems and Integral Equations, Cambridge University Press, Cambridge, 2000.

[29] W. Rundell, Recovering an obstacle and its impedance from Cauchy data, Inverse Problems, (24)2008, 045003, (22pp). 